\title{
CARACTERIZACIÓN GEOMORFOLÓGICA DE LOS RÍOS DE GALICIA MEDIANTE UNIDADES LITOTOPOGRÁFICAS
}

\author{
Jesús Horacio García \\ Dpto. Xeografía, Fac. de Xeografía e Historia, Univ. de Santiago de Compostela \\ Pr. da Universidade, 1, 15782, Santiago de Compostela, España \\ horacio.garcia@usc.es
}

Resumen: Se presenta una definición de unidades litotopográficas para Galicia que permite la segmentación de ríos y, a su vez, un modo de clasificar los sistemas fluviales. Dichas unidades se definen como un sector de igual topografía y litología, por lo tanto, de áreas en las que los procesos ecológicos del río responden a comportamientos similares. La definición de unidades litotopográficas se ha desarrollado a partir del análisis cartográfico, empleando para ello técnicas multivariantes para las variables topografía (altitud, rugosidad y pendiente) y litología. En una etapa posterior, se han medido diversos parámetros geomorfológicos (pendiente del cauce, radio hidráulico, ratio anchura/profundidad del cauce, mediana del tamaño del sedimento y potencia específica) en $23 \mathrm{sec}-$ tores del río Cabe (Lugo) que engloban diversas unidades litotopográficas. Los resultados muestran que los descriptores geomorfológicos manejados se encuentran para cada unidad litotopográfica dentro de una horquilla de valores muy parejos, lo que permite su identificación como unidades internamente homogéneas y claramente diferenciables de las otras. La zonificación del territorio en unidades litotopográficas facilita la aplicación de medidas de gestión y la comparación de sistemas fluviales.

Palabras clave: unidad litotopográfica, geomorfología fluvial, caracterización hidromorfológica, clasificación fluvial, Directiva Marco del Agua (2000/60/CE), Galicia.

Abstract: A lithotopo unit definition proposal is made for Galicia. A lithotopo unit will be defined as a sector with equal topography and lithology,

Recibido: 21-02-13. Aceptado: 31-05-13. 
therefore, as an area where the ecological processes display similar behaviours. This definition allows the segmentation of rivers as well as the classification of fluvial systems. The lithotopo units have been developed from cartographic analysis, using multivariate techniques for two variables: topography (elevation, slope and roughness) and lithology. Next, several geomorphic parameters (channel slope, hydraulic radius, width/depth ratio of the channel, sediment median size and specific power) were measured in twenty-three working sectors along the Cabe River (Lugo, NW Spain) which encompass different lithotopo units. The results show that the geomorphic descriptors for each lithotopo unit vary within a similar range of values. This range is enough to allow their identification as internally homogeneous and mutually distinguishable units. The territorial mapping into lithotopo units makes it easier both to implement management measures and to make comparisons between fluvial systems.

Keywords: lithotopo unit, fluvial geomorphology, hydromorphological characterization, fluvial classification, Water Framework Directive (2000/ 60/CE), Galicia (NW Iberian Peninsula).

\section{Introducción}

Un sistema fluvial lo forma un amplio conjunto de elementos con interacciones múltiples a diferentes escalas espaciales y temporales y, por tanto, con un comportamiento no lineal (Knighton, 1998). De entre estos factores, el geomorfológico constituye uno de los ejes centrales sobre el que se sustentan todos los demás (Downs y Brookes, 1994; Newson et al., 1998) y, entre ellos, destaca la componente biológica (Frissell et al., 1986), empleada en numerosos estudios de valoración y restauración fluvial (Palmer et al., 2010). Desde una óptica jerárquica, todo sistema fluvial está condicionado por las componentes climática y, muy especialmente, estructural; esto es, la litología y la tectónica (Montgomery y Buffington, 1998; Brierley y Fryirs, 2005). Ambos elementos, interrelacionados a lo largo del tiempo, favorecen la presencia de un tipo de relieve concreto y de un régimen hidrológico y sedimentológico determinado, lo que, a su vez, está en relación con los procesos y formas presentes en un sistema fluvial (Brussock et al., 1985).

Dado que la dinámica geomorfológica interviene en la construcción de un tipo de hábitat concreto (Hawkins et al., 1993), la comprensión ecológica de todo sistema fluvial necesita per se, y en primer orden, comprender la dimensión geomorfológica (Fryirs y Brierley, 2012). En este sentido, las características ecológicas del cauce son reflejo de las características geomorfológicas (litología y topografía) y climáticas, así como las derivadas de ambas, que encierra la cuenca vertiente (Petts y Amoros, 1996). No obstante, la complejidad de estudio de un sistema fluvial supone que no siempre sea fácil deslindar causa y efecto, o variables dependientes de independientes (Fernández et al., 2012). 
Los ríos tradicionalmente han sido clasificados, valorados o gestionados en función de criterios hidrológicos, químicos o biológicos (Illies y Botosaneanu, 1963; Pennak, 1971; Hem, 1985; Naiman, 1998; Wright et al., 1998; Poff et al., 1997; Angelier, 2002; Brooks et al., 2012), relegándose la geomorfología a un segundo plano (Thorne, 1997; Schmitt, 2001). En este sentido, la aprobación de la Directiva Marco del Agua (2000/60/CE) en el año 2000 (en adelante, DMA), marca un importante punto de inflexión. Por un lado considera la geomorfología como un elemento clave en la determinación del estado de los sistemas fluviales y en la búsqueda de soluciones. Y, por otro lado, esta nueva Directiva es de obligado cumplimiento para los Estados miembros. No obstante, la implementación de estas normativas, así como el rico legado existente en forma de clasificaciones de cursos fluviales desde criterios geomorfológicos (véase al respecto las recopilaciones bibliográficas confeccionadas por Mosley, 1987; Kondolf, 1995; Kondolf et al., 2003), no han impedido la existencia de un vacío metodológico en la tipificación y caracterización hidrogeomorfológica (Newson, 2012).

La clasificación de los ríos representa una de las recomendaciones de la DMA y establece que ha de desarrollarse con base en diferentes variables y atributos, organizados jerárquicamente de menor a mayor escala (ecorregión - cuenca vertiente - segmento fluvial - hábitat fluvial - comunidades biológicas), de forma que se garantice el establecimiento de unas condiciones de referencia para cada río de cada Estado miembro (González del Tánago y García de Jalón, 2006). El grupo de descriptores del Sistema A de la DMA es de carácter obligatorio (región ecológica, altitud, tamaño cuenca vertiente y geología) y busca contextualizar el río a escala regional y de cuenca. El Sistema B propone varios descriptores optativos que interactúan a diferentes escalas espaciales y ayudan en el grado de discriminación. La ecorregión agrupa áreas ecológicamente homogéneas a gran escala (Bailey, 2005). Según el Anexo XI de la DMA, Galicia pertenece a la región ibérico-macaronésica. Rivas-Martínez et al. (2004) sitúan a la Comunidad dentro del tipo 4 a (Subprovincia Cántabro-Atlántica) en su mapa biogeográfico de Europa (escala 1:6.000.000). Por su parte, Lara et al. (2007) la encuadra en la región de Galicia, según su mapa de vegetación de ribera en el norte peninsular. A escala de más detalle, Rodríguez-Guitián y Ramil (2007) han establecido para Galicia diversas cartografías ecológicas y bioclimáticas de gran interés para zonificar internamente las cuencas como un primer nivel de caracterización.

El presente trabajo surge como respuesta a dos interrogantes: ¿son necesarias las clasificaciones? y, en segundo término, ¿identifican las escalas espaciales 'ecorregión' y 'cuenca vertiente' áreas en las cuales los procesos y formas ecológicas se comporten de modo similar? Para el primer planteamiento se considera el "sí" como respuesta (Naiman et al., 1992; Juracek y Fitzpatrick, 2003). Las clasificaciones permiten una mejor compresión de las formas y procesos estudiados, promoviendo al mismo tiempo la teorización y empirismo de leyes que expliquen las diferencias de los objetos clasificados (Goodwin, 1999). Trasladado al ámbito fluvial, Kondolf et al. (2003) consideran dos los objetivos fundamentales de las clasificaciones. Por una parte, la compresión cien- 
tífica de cómo funcionan los ríos y de qué modo se pueden, por agrupación, homogeneizar en clases y, por otra parte, han de servir como guía técnica en la toma de decisiones sobre la ordenación o restauración fluvial.

Respecto a la cuestión de si son capaces las dos escalas espaciales de regionalización de la DMA de explicar, de forma consistente, espacios hidrogeomorfológicamente diferenciados, se considera que la zonificación en ecorregiones es de insuficiente grado de detalle como para establecer comportamientos hidrológicos y geomorfológicos comunes, congregándose en un mismo espacio tipologías muy dispares (Hawkins y Norris, 2000). La organización en cuencas vertientes de la DMA trata de dar respuesta a las características de caudalosidad (tamaño), de escorrentía (geología) y pluviotérmicas (altitud). Sin embargo, condiciones hidrológicas y escorrentía no se ciñen exclusivamente a la geología, sino que dependen también de otros factores no recogidos por la DMA como los usos del suelo, la vegetación o el tipo edafológico. Del mismo modo, los límites de la cuenca no tienen por qué coincidir con los límites de unidades litológicas homogéneas, ni existe razón para que se distingan procesos geomorfológicos y hábitats característicos en función del tamaño. Además la propuesta divisiva de la DMA para la geología es de muy poco detalle con lo que, al igual que sucede con las ecorregiones, en una misma cuenca vertiente o tipología geológica, pueden coexistir tipos de ríos con marcadas diferencias (Omernik y Bailey, 1997).

\section{Encuadre territorial}

La cartografía de las unidades litotopográficas se ha desarrollado a nivel regional para toda la Galicia peninsular ( $\sin$ tener en consideración las islas, $0,08 \%$ de la superficie total), mientras que el testado o validación del mapa se ha llevado a cabo para la cuenca lucense del río Cabe (figura 1).

La cuenca del Cabe tiene una superficie de $733 \mathrm{~km}^{2}$ (2,5\% de la superficie de Galicia), una altitud media de $510 \mathrm{~m}$, con un rango altitudinal de $1.171 \mathrm{~m}(1.299 \mathrm{~m}-128$ m). La pendiente media es del $16,7 \%$. La estructura fisiográfica de la cuenca se caracteriza por la existencia de una gran depresión central envuelta por un reborde montañoso continuo que se acentúa hacia su flanco nororiental. El río Cabe tiene una longitud de 59,6 km (en la figura 1 se marca en línea discontinua lo que se consideró para este trabajo como cauce principal y, en línea continua, según el Mapa Topográfico Nacional 1:25.000), con un caudal medio de $5,17 \mathrm{~m}^{3} / \mathrm{s}$ (serie 1975-2009) en la estación foronómica de Rivas Altas para una cuenca vertiente de $353 \mathrm{~km}^{2}$. Destacan como principales afluentes por su margen derecha los ríos Mao $(32,8 \mathrm{~km})$ y Cinsa $(22,0 \mathrm{~km})$, siendo el Saa $(20,2 \mathrm{~km})$ el río más relevante por la margen izquierda.

La elección del Cabe como cuenca de análisis de las unidades litotopográficas se justifica por su diversidad de ambientes litológicos y topográficos, lo que a la postre se traduce en unidades litotopográficas con ambientes geomorfológicos de elevado contraste. 

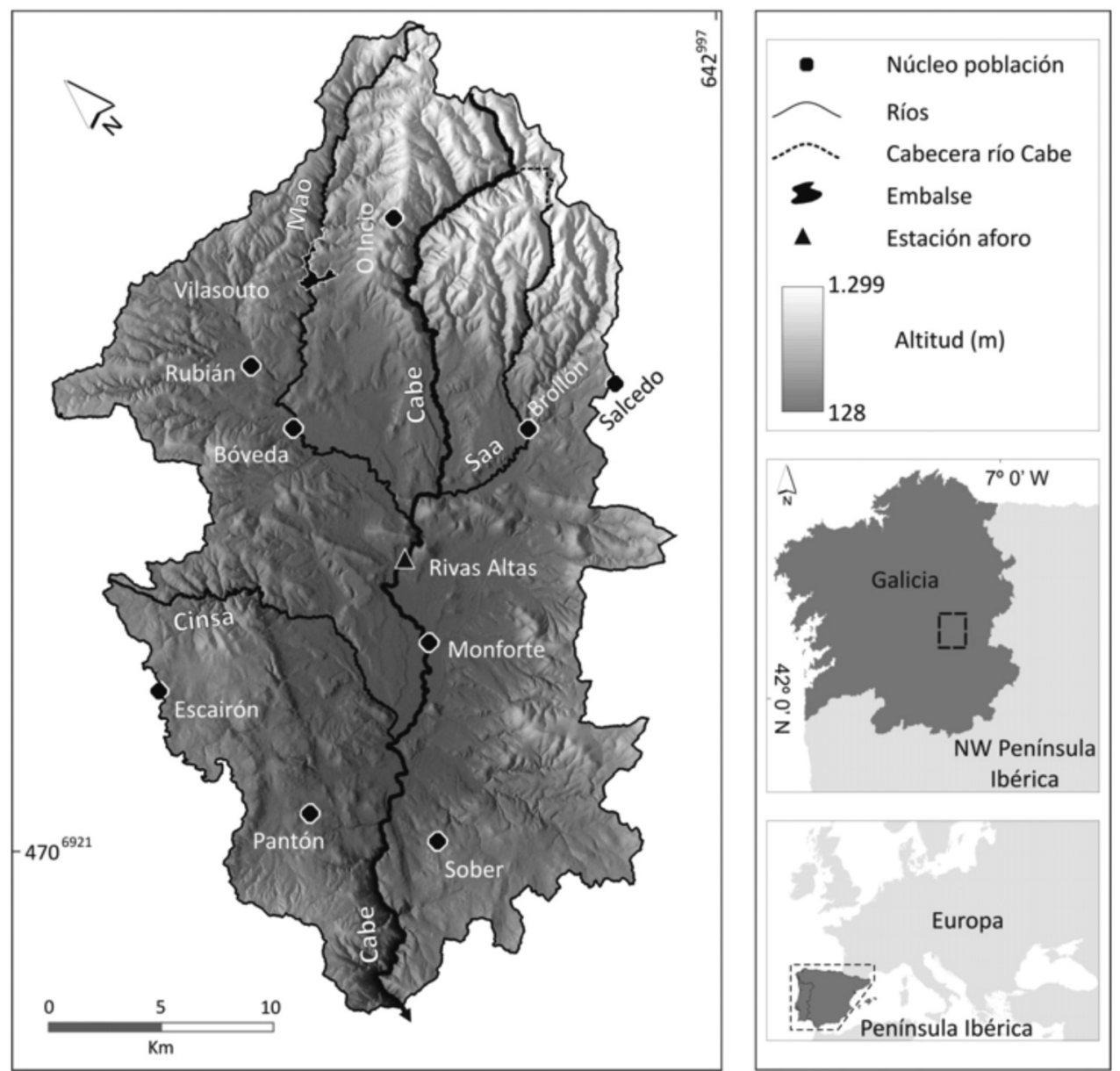

Figura 1. Localización del área de estudio. Cuenca del río Cabe, Lugo.

\section{Objetivos y metodología}

El propósito de este estudio es la identificación de unidades espaciales con características de relieve y litología comunes y su representación cartográfica en toda la superficie de Galicia continental. Este objetivo principal se puede dividir en dos más concretos: i) establecer una metodología de definición e identificación de las unidades litotopográficas, y ii) contrastar mediante variables de uso frecuente en trabajos de geomorfología fluvial -algunas de ellas pertenecientes al Sistema B de la DMA-, la validez de las unidades cartográficas como criterio de segmentación de sectores fluviales. 
El primero de ellos se ha definido para toda Galicia. El segundo se ha puesto en práctica, a modo de ensayo, en la cuenca del río Cabe.

Así, con este estudio se propone elaborar un nuevo nivel jerárquico de organización espacial que unifique territorios con similar topografía y litología y, por tanto, con procesos geomorfológicos análogos (Montgomery, 1996). Es lo que el último autor citado denominó unidades litotopográficas (en adelante, ULT). Estas unidades se conciben como una herramienta flexible que ayuda a la comprensión de la estructura geomorfológica y, en consecuencia, también ecológica, de los sistemas fluviales (Kondolf, 1995; Poole, 2002). Con el nuevo panorama jerárquico propuesto, la ecorregión contextualiza a los ríos en grandes dominios biogeográficos, mientras que la cuenca vertiente (caracterizada por su tamaño) se estructura internamente según las ULT (Montgomery, 1996).
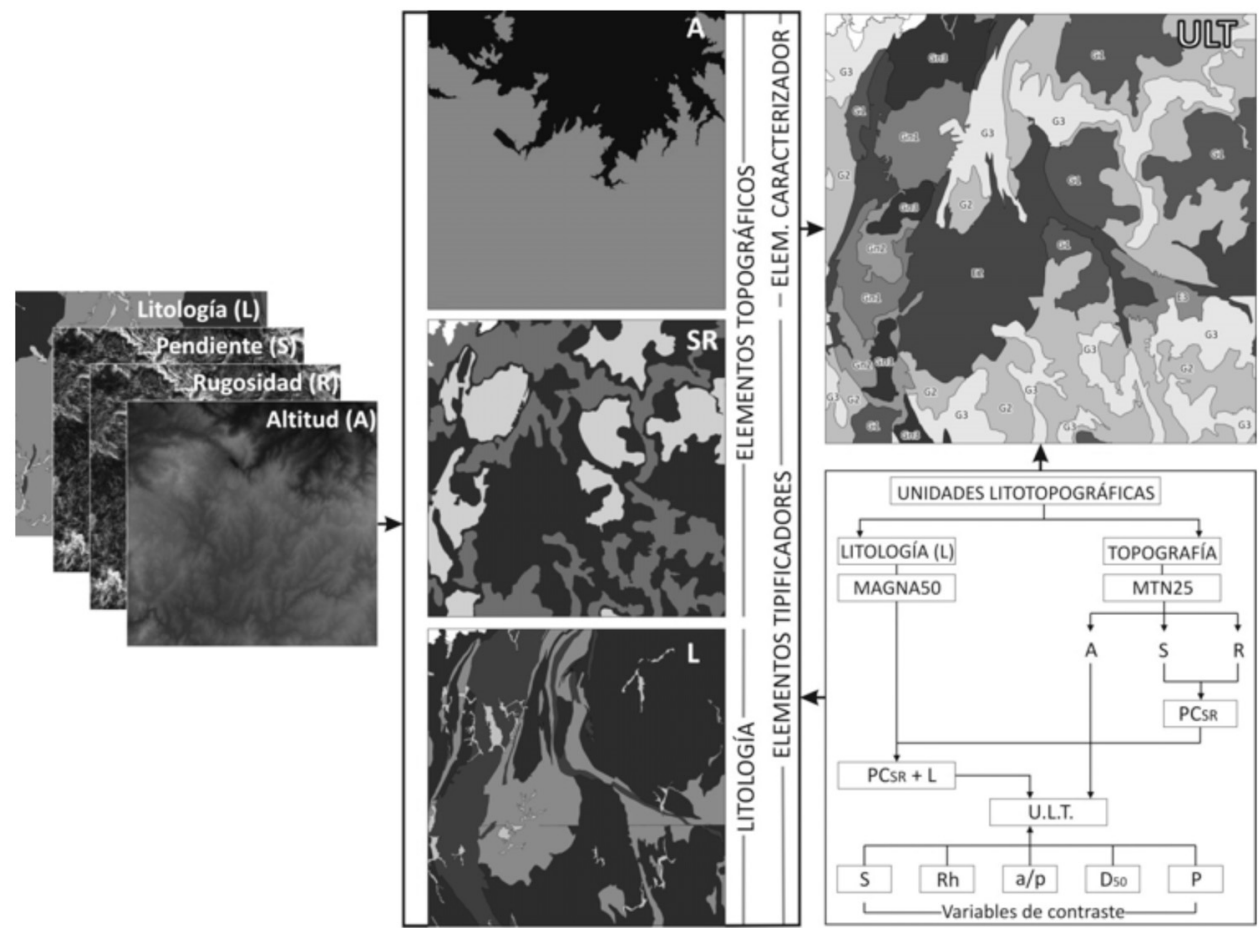

Figura 2. Esquema metodológico seguido para el desarrollo cartográfico de las ULT y su posterior validación. En las variables de contraste: S (pendiente), Rh (radio hidráulico), $\mathrm{a} / \mathrm{p}$ (ratio anchura / profundidad del cauce), $\mathrm{D}_{50}$ (mediana del tamaño del sedimento),

$\mathrm{P}$ (potencia específica). $\mathrm{PC}_{\mathrm{SR}}$ es la componente principal $(\mathrm{PC})$ de la agrupación por cluster entre pendiente $(S)$ y rugosidad $(R)$ del territorio. 
El procesado cartográfico, basado en el principio de regionalización geográfica (Herod, 2003), se ha desarrollado con apoyo de los Sistemas de Información Geográfica (en adelante, SIG). Se ha trabajado en ETRS89 29N, con una resolución de píxel de 50x50 m (escala 1:250.000) y una unidad mínima cartografiable (UMC) de $10 \mathrm{~km}^{2}$ (de acuerdo con la DMA, este es el tamaño mínimo para la caracterización de los tipos de masa de agua superficial, según el criterio de superficie de la cuenca de alimentación). Se ha comprobado que, a escala regional, trabajar con resoluciones de más detalle o fijar como UMC zonas menores a $10 \mathrm{~km}^{2}$ fragmenta en exceso el territorio, sin obtener necesariamente unos mejores resultados.

Como técnicas de análisis estadístico se ha utilizado un análisis ANOVA, para determinar la similitud entre las variables de contraste y si este parecido está relacionado con alguna ULT concreta; técnicas multivariantes factoriales y de clasificación, para observar si los puntos de muestreo de cada ULT del río Cabe presentan características comunes; y correlaciones entre variables mediante los coeficientes de Spearman y Pearson. El nivel de confianza aplicado para la significancia estadística ha sido del 5\%.

Para la definición de las unidades litológicas se empleó la cartografía geológica de la serie MAGNA (escala 1:50.000), desarrollada por el IGME en su versión digital (shapefile). En el proceso de depuración al que ha sido sometida la capa de litología se encontraron 36 errores topológicos (gaps) y varios errores de asignación. Ambos fueron subsanados desde el SIG con diversas técnicas geoespaciales.

Los grupos litológicos se han establecido buscando que alberguen la mayor homogeneidad posible frente al comportamiento geomorfológico de un río, por tanto, considerando la petrología y otros factores físico-químicos influyentes en el modelado fluvial. Esto ha supuesto una reorganización de la cartografía litológica de acuerdo a dos factores: el contenido en sílice $\left(\mathrm{SiO}_{2}\right)$ y el comportamiento mecánico de la roca frente a la erosión (rocas isótropas y rocas anisótropas). El resultado es la división de Galicia en 5 grandes dominios litológicos: i) granitoides (granitoides alcalinos, granitoides calcoalcalinos, anfibolitas, gabro), ii) pizarras y cuarcitas (pizarra, cuarcita, caliza), iii) gneis (gneis, periodita, serpentinita, eclogita, granulita), iv) esquistos y v) depósitos (depósitos terciarios, depósitos cuaternarios). Cabe apuntar que según sea el nivel de detalle del estudio, es decir, en función de la escala de trabajo, será necesario descender a niveles de más detalle litológico. Incluso, para el presente estudio, la propuesta de 5 unidades litológicas podría presentar, como alternativa, otra que tuviese un sexto grupo denominado "rocas máficas y ultramáficas" que agrupase a: granulitas, anfibolitas, eclogitas, gabros e incluso peridotitas y serpentinitas.

Frente a esta fusión de las unidades litológicas con características similares, existe también un elevado número de manchas con escasa representación espacial, y sin una asignación litológica del todo exacta en alguno de los grupos litológicos preestablecidos. La solución cartográfica para estos casos ha consistido en acoplar cada pequeña mancha a la unidad litológica con la que comparta la mayor parte de su perímetro. 
Respecto de la información topográfica requerida para el estudio, esta ha sido suministrada por el Instituto Geográfico Nacional (IGN) en mallas de puntos (ASCII) a escala 1:25.000 (C Instituto Geográfico Nacional de España, 2008). La calidad de los datos se validó comprobando para diferentes interpoladores (Spline, Kriging, Inverse Distance Weighted, etc.) el grado de incertidumbre y exactitud, calificándose los mismos como de alta calidad (Horacio, 2010).

En el establecimiento de unidades homogéneas de relieve a partir del modelo digital del terreno (en adelante, MDT) (Weibel y Heller, 1991; Felicísimo, 1994), se hizo necesario un doble planteamiento: qué variables se emplean y de qué modo se agrupan. Para la primera cuestión se ha optado por el uso de tres indicadores de base común pero de información sustancialmente diferente (Figura 2): altitud, pendiente y rugosidad (Barredo y Bosque-Sendra, 1996; Wasson et al., 2002). La altitud se ha considerado un valor secundario, más caracterizador que tipificador (Figura 2). La correlación de Spearman $(\rho)$ entre las variables que dan forma al relieve (pendiente y rugosidad) y la altitud es mínima $(\rho=0,08)$, pero no entre pendiente y rugosidad $(\rho=0,89)$, con lo que, por ejemplo, a muy diferentes intervalos altitudinales pueden aparecer idénticos paisajes topográficos (llanura aluvial, superficie de erosión, meseta, etc.) De este modo, mientras que la rugosidad y la pendiente se han empleado para zonificar el relieve (parámetros tipificadores), la altitud se ha utilizado como un factor caracterizador que puede ayudar en la comprensión de las características del río, pero, dada la escasa altura del relieve gallego, más desde un punto de vista biológico que geomorfológico (RodríguezGuitián y Ramil, 2007).

El valor altitudinal procede directamente del MDT, mientras que la pendiente y la rugosidad derivan de él. La altitud al considerarse como un parámetro caracterizador, no ha sido incluida en la Figura 3. La pendiente se ha obtenido, para cada celda de la capa ráster del MDT, calculando la tasa máxima de cambio de valores z sobre un plano de vecindad 3x3 alrededor de dicha celda procesamiento (Burrough y McDonnell, 1998). En un punto dado sobre una superficie $\mathrm{z} f(\mathrm{x}, \mathrm{y})$, la pendiente $(\mathrm{S})$ se define como una función de los gradientes en $\mathrm{x}$ e y:

$$
S=\arctan \sqrt{f_{x}^{2}+f_{y}^{2}}
$$

Sobre el procedimiento de cálculo del parámetro rugosidad no existe un consenso (Evans, 1972; Balce, 1987; Felicísimo, 1994), lo que a su vez es reflejo de un marco teórico muy variado. De forma conceptual se podría definir la rugosidad como la medida de la irregularidad o accidentalidad del terreno, es decir, su heterogeneidad (Pike et al., 2008). La variedad de propuestas metodológicas es alta (Peuker y Douglas, 1975; Zenverbergen y Thorne, 1987; Moore et al., 1991; Jenness, 2004; Sappington et al., 2007). Para el presente trabajo se ha obtenido la rugosidad por píxel a partir de la propuesta de Riley et al. (1999), cuya expresión matemática es:

$$
I R T_{r c}=\sqrt{\sum_{i=r-1}^{r+1} \sum_{j=c-1}^{c+1}\left(e_{i, j}-e_{r, c}\right)^{2}}
$$


Donde $e$ es la altitud del píxel correspondiente a la fila $r$ (row) y la columna $c$ (column) e IRT el índice de rugosidad topográfica (Topographic Ruggedness Index) de Riley et al. (1999). Para el análisis espacial de este parámetro se ha usado un script de tipo 'aml' (Arc macro languaje) ejecutado en ArcInfo Workstation (ESRI) e, igualmente que la pendiente, con una kernel $3 \times 3$.

La segunda de las cuestiones a dilucidar, es decir, cómo se agrupan las variables, ha consistido en una clasificación multivariante no supervisada (análisis de conglomerados, cluster), en cuanto que, a priori, se desconocen las dimensiones y, en menor medida, ubicaciones de las unidades homogéneas de la zona de estudio. Como medida de disimilitud se ha utilizado un algoritmo local de búsqueda por escalamiento (bill-climbing), implementado en el software SAGA (System for Automated Geoscientific Analyses) 2.0.8. (Rubin, 1967). El tipo de correlación entre las variables altitud, pendiente y rugosidad, hizo necesario estructurar parte de la información en componentes principales.

\subsection{Variables de contraste y validación de las ULT}

El hecho de que un eje fluvial que atraviese una determinada ULT presente un comportamiento hidromorfológico distinto a otra, se contrastó mediante trabajo de campo en 23 puntos de muestreo de la cuenca del río Cabe (Lugo). Estos lugares de muestreo han sido seleccionados según tres criterios: primero, que todo el río estuviese representado en su recorrido longitudinal, por tanto, con una distancia similar entre tramos. En segundo lugar, también se fijaron como puntos de muestreo aquellos enclaves litológicos representativos que no fueron recogidos con el anterior procedimiento. En último término, se hicieron medidas justo aguas arriba y aguas abajo de la unión al río Cabe de los afluentes Mao, Saa y Cinsa. En el tramo bajo del río (sector granitoide) las complicaciones de acceso han reducido la densidad de puntos de muestreo respecto al resto de tramos. Los puntos de muestreo más próximo son Ca14 y Ca15, a $639 \mathrm{~m}$ el uno del otro y, los más alejados, Ca19 y Ca20 (7,0 km), entre los cuales se ubica la ciudad de Monforte de Lemos (ver Figura 3), con el río parcialmente canalizado. La longitud media del tramo es de 2,5 km, con una desviación estándar de 1,7 km.

En cada punto de muestreo se midieron las siguientes variables: dos que definen la hidráulica a nivel de caudal generador (en muchas situaciones asociadas con el estado bankfull) que describan forma y tamaño del cauce (ratio anchura/profundidad máxima, "a/p", y radio hidráulico (área/perímetro mojado), "Rh"), una sedimentaria $\left(\mathrm{D}_{50}\right)$, y una cuarta y quinta referidas a la energía del río, expresadas mediante la pendiente local (S) y, en especial, por la potencia específica (P) (Bagnold, 1960), interpretada por medio de la siguiente expresión:

$$
\mathrm{P}=\left(\mathrm{mva} * \mathrm{~g} * \mathrm{Q}_{\mathrm{b}} * \mathrm{~S}\right) / \mathrm{w}
$$


donde $P$ es la potencia específica (watios $/ \mathrm{m}^{2}$ ), $m v a$ la masa volumétrica del agua (1.000 $\left.\mathrm{kg} / \mathrm{m}^{3}\right), g$ la aceleración de la gravedad $\left(9,8 \mathrm{~m} / \mathrm{s}^{2}\right), Q_{b}$ el caudal bankfull $\left(\mathrm{m}^{3} / \mathrm{s}\right), S \mathrm{la}$ pendiente local $(\mathrm{m} / \mathrm{m})$ y $w(\mathrm{~m})$ la anchura bankfull o generadora.

El caudal generador marca el nivel de referencia más influyente en la geomorfología y ecología de un cauce (Martín-Vide, 2002; Ollero, 2011). Este caudal se vincula con la geometría hidráulica (Leopold y Maddock, 1953) y el transporte de sedimentos del río (Watson et al., 1999). Por consiguiente, la elección de las 5 variables de contraste está orientada a representar, en unas pocas funciones empíricas, la mayor comprensión de la morfología fluvial de un río. El tamaño de la geometría hidráulica queda representado por el radio hidráulico (Rh), mientras que la relación a/p se asocia con la forma del cauce. El percentil $50\left(\mathrm{D}_{50}\right)$ informa del tamaño medio del material removible. La pendiente del cauce (S) señala la energía potencial puesta a disposición del flujo por unidad de longitud recorrida. La potencia específica es indicativa de la competencia de la corriente para movilizar sedimentos, por lo que es un parámetro muy útil para comparar unos ríos con otros o bien diferentes tramos de un mismo sistema fluvial (Schmitt et al., 2007).

Las variables hidráulicas han sido medidas con una estación total Leica TC 307. La sección topográfica siempre se ha prolongado más allá de lo que en campo consideramos se elevaba la cota del caudal generador y, de este modo, asegurarnos su inclusión. Entre una medida topográfica y otra nunca se ha excedido el 5\% de la amplitud de la sección ni se han hecho a intervalos regulares, sino en función de la irregularidad de dicha sección. Para los cálculos geométricos de los datos recogidos en campo se ha utilizado el software WinXSPRO 3.0 (Hardy et al., 2005) del Stream Systems Technology Center de EE.UU. La pendiente del cauce se ha obtenido midiendo la altura de la lámina de agua en $50 \mathrm{~m}$ de cauce, $25 \mathrm{~m}$ aguas arriba y $25 \mathrm{~m}$ aguas abajo del punto de muestreo.

La caracterización sedimentaria de cada punto de muestreo se ha hecho mediante un análisis granulométrico superficial, siguiendo las pautas fijadas por Wolman (1954) en la denominada como técnica de "conteo superficial" (véase también Church et al., 1987; García, 1994; Kondolf et al., 2003). Como herramienta de apoyo para el conteo de material inferior al tipo large cobble (Wentworth, 1922), se ha empleado un gravelómetro de 13 aperturas (en mm): 2, 2,83, 4, 5,66, 8, 11,3 16, 22,6, 32, 45,3, 64, 90,5 y 126. En aquellos sectores de análisis con presencia de arenas y finos, se tomó una muestra aparte para tamizar y pesar por separado (se ha considerado como material fino todo aquel menor de 5,66 mm). Como norma general, se acepta que si el 10\% de los sedimentos muestreados salen de arena o fino ya no se hace conteo, sino que se separan las muestras por tamaños. El número de muestras siempre ha sido superior a 100 salvo en los puntos de muestreo Ca04, Ca08, Ca10, Ca20, Ca25, Ca26, Ca29, en los que se contabilizaron respectivamente 40, 90, 75, 80, 70 y 75 clastos. El menor número de elementos en estos puntos se debe a que parte del lecho es de roca, lo que reduce la proporción de material aluvial. 


\section{Resultados}

\subsection{Cartografía de ULT}

Del resultado cartográfico correspondiente con la litología, se ha observado que la pequeña representación espacial de algunas unidades litológicas supone que carezcan de sentido cartográfico para la escala de trabajo desarrollada, aún a sabiendas de que son sectores con una dinámica geomorfológica propia. Un ejemplo destacado son las rocas filonianas (ejemplo paradigmático: dique de cuarzo de Boqueixón cortado por el río Ulla), con la singularidad de que, además, existen rocas filonianas de naturaleza granítica (aplita, pegmatita, pórfido), gabroica (diabasa, dolerita, pórfido) o monominerálica (cuarzo), con un comportamiento extremadamente distinto, aún perteneciendo al mismo grupo. Igualmente, dentro de un mismo dominio litológico pueden darse importantes contrastes que deriven en subgrupos diferenciados -en rocas graníticas, el grado de fracturación (diaclasas) o de arenización (xabre en gallego)-.

En el análisis previo al desarrollo cartográfico de las ULT (Figura 3), se ha analizado la correlación y covariancia) entre los parámetros altitud y pendiente $(0,26 / 176,3)$, altitud y rugosidad $(0,27 / 263,3)$, y pendiente y rugosidad $(0,89 / 22,1)$. La elevada correlación entre las variables encargadas de estructurar el relieve (pendiente y rugosidad), hizo aconsejable un previo análisis de componentes principales que asegurase una alta separabilidad en clases de los datos correlacionados y, de este modo, proceder a la clasificación multivariante (análisis de cluster) con variables sin interdependencia (Felicísimo, 1994).

La clasificación no supervisada del primer componente principal pendiente-rugosidad $\left(\mathrm{PC}_{\mathrm{SR}}\right.$ 01: 94,3\% de la varianza), se realizó inicialmente con un número de 8 grupos, aunque esta cifra inicial se ha ido reajustando, bajo criterio experto, hasta fijar 4 como el número de unidades de relieve o topográficas más apropiado para la escala de trabajo empleada. La combinación de la cartografía de estas 4 unidades de relieve con la capa de litología ( 5 tipos), arroja 18 ULT con diferentes características de altitud, pendiente, rugosidad y litología (ver Figura 3 y Tabla 1). Las ULT se nombran indicando mediante una letra el tipo litológico dominante (D, E, G, Gn, PC), seguido de un número que señala el tipo topográfico al que pertenece $(1,2,3$, 4). Las cuatro unidades topográficas establecidas presentan unos valores de pendiente y rugosidad concretos, desde los terrenos menos accidentados (tipo 1), hasta otros con pendientes acusadas y predominio de la verticalidad (tipo 4). En la Tabla 1 se aprecia como para las unidades topográficas tanto la altitud, como pendiente y rugosidad, incrementan desde la unidad 1 hasta la 4 . Por ejemplo, la unidad topográfica 1 tiene una pendiente media de $2,0^{\circ}$, por $4,9^{\circ}$ de la unidad $2,10,0^{\circ}$ de la 3 y $20,3^{\circ}$ de la unidad 4 . Las unidades litológicas también presentan diferentes valores medios de altitud, pendiente y rugosidad, si bien, no siguen ninguna secuencia organizada. 

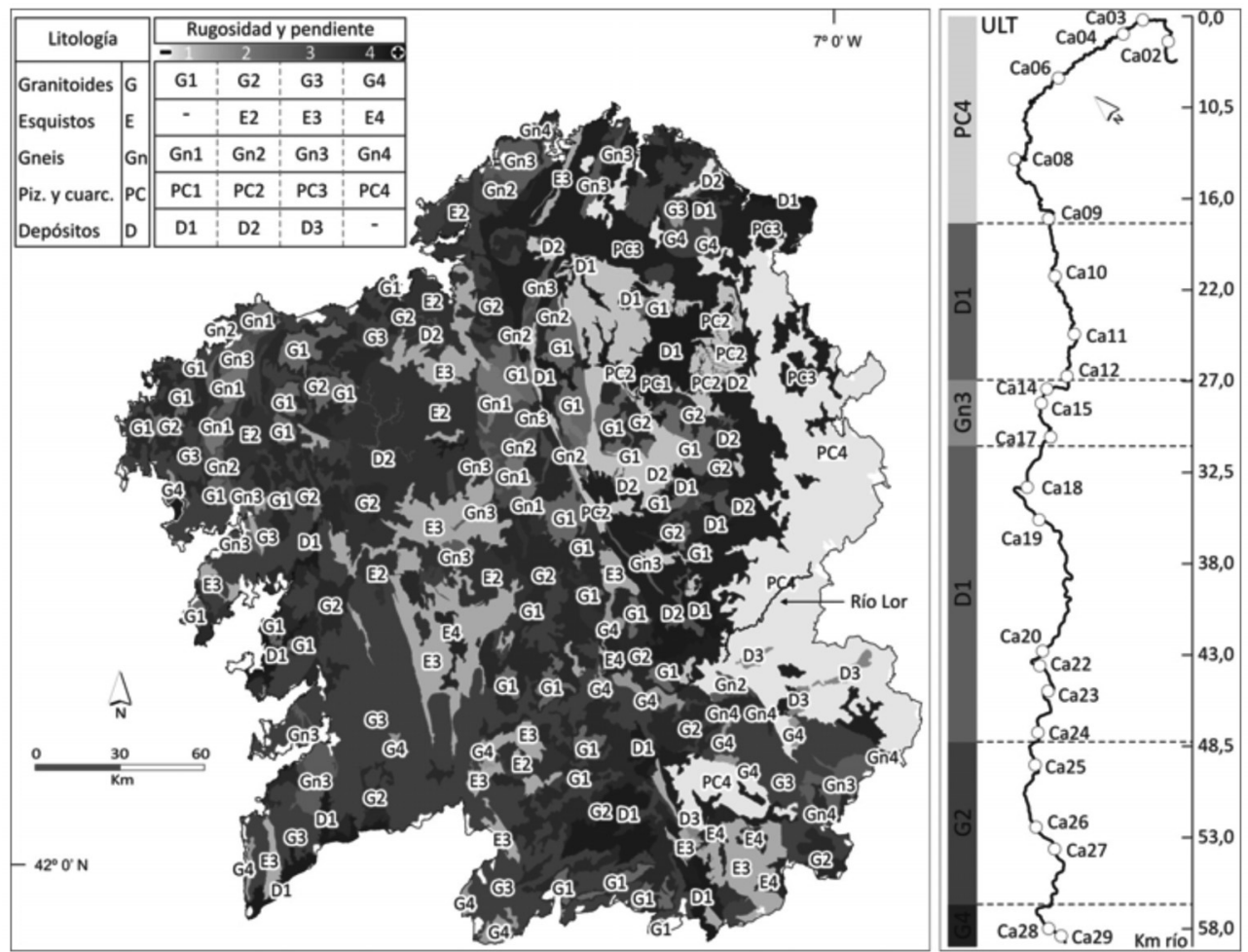

Figura 3. Propuesta de ULT para Galicia y localización de los puntos de muestreo en el río Cabe.

Desde un punto de vista de ocupación espacial, más del 75\% de Galicia pertenece a las ULT (ver Figura 3): G3 (22,0\%), G2 (15,3\%), PC3 (11,5\%), PC4 (11,4\%), E2 (8,8\%) y E3 (7,3\%). En el extremo opuesto están aquellas que ostentan menos de un 1\% de presencia: PC1, D3, Gn4, D2 y E4. Por unidades litológicas, casi la mitad del territorio está comandado por el grupo granitoide (40,4\%), seguido de pizarras y cuarcitas (26,6\%). Los gneis con un 6,7\% de representación, ocupan el último lugar. En cuanto a las unidades topográficas, los tipos $2(30,9 \%)$ y $3(43,8 \%)$ representan al 75\% de Galicia. A una distancia mucho mayor está los tipos extremos, aquellos relieves más abruptos (tipo 4 con un 13,9\% de ocupación) y los más planos (tipo 1 con un 11,5\%). Las unidades litológicas pueden abarcar diferentes dominios topográficos y, viceversa, un mismo tipo de relieve es posible que aparezca en diferentes litologías (Figura 3).

Se ha observado que en la categoría litológica depósitos (D) y en la de tipo de relieve de categoría 1, los valores más elevados de altitud, pendiente y rugosidad se alejan mucho respecto a la media. Este tipo de "errores conocidos" se producen por un desajuste escalar con el tamaño de celda manejado $(50 \times 50 \mathrm{~m})$. La matriz de cálculo 
Tabla 1. Características topográficas de las ULT, unidades litológicas y unidades topográficas establecidas para Galicia, donde: $\mu$ (media), ma (mediana), sd (desviación estándar) y cv (coeficiente de variación), D (depósitos), E (esquistos), G (granitoides), Gn (gneis) y PC (pizarra y cuarcita)

\begin{tabular}{|c|c|c|c|c|c|c|c|c|c|c|c|c|c|}
\hline \multirow{2}{*}{$\begin{array}{l}\text { Tipos } \\
\text { ULT }\end{array}$} & \multirow{2}{*}{$\begin{array}{c}\text { Superf. } \\
(\%)\end{array}$} & \multicolumn{4}{|c|}{ Altitud (A) } & \multicolumn{4}{|c|}{ Pendiente (S) } & \multicolumn{4}{|c|}{ Rugosidad (R) } \\
\hline & & $\mu$ & $\mathrm{ma}$ & sd & $\mathrm{CV}$ & $\mu$ & $\mathrm{ma}$ & sd & $\mathrm{CV}$ & $\mu$ & $\mathrm{ma}$ & $\mathrm{sd}$ & $\mathrm{CV}$ \\
\hline D1 & 5,2 & 373,9 & 409 & 199,1 & 53,2 & 1,6 & 1 & 2,2 & 60,8 & 2,6 & 2 & 2,9 & 75,8 \\
\hline D2 & 0,7 & 352,5 & 384 & 157,2 & 44,6 & 5,0 & 4 & 4,2 & 80,5 & 7,7 & 7 & 5,1 & 90,5 \\
\hline D3 & 0,3 & 412,4 & 408 & 106,6 & 25,8 & 7,7 & 7 & 6,0 & 90,7 & 11,8 & 11 & 7,7 & 93,4 \\
\hline E2 & 8,8 & 330,1 & 332 & 171,2 & 51,9 & 4,5 & 4 & 3,1 & 88,5 & 6,8 & 6 & 3,7 & 88,8 \\
\hline E3 & 7,3 & 491,9 & 470 & 262,8 & 53,4 & 10,4 & 10 & 5,7 & 96,2 & 15,4 & 14 & 7,1 & 90,9 \\
\hline E4 & 0,8 & 709,8 & 698 & 242,1 & 34,1 & 18,8 & 19 & 7,5 & 101,2 & 28,4 & 28 & 9,0 & 98,7 \\
\hline G1 & 5,1 & 447,1 & 475 & 180,3 & 40,3 & 2,8 & 2 & 2,2 & 70,8 & 4,3 & 4 & 2,6 & 92,6 \\
\hline G2 & 15,3 & 461,5 & 468 & 259,3 & 56,2 & 5,6 & 5 & 3,8 & 88,7 & 8,4 & 8 & 4,6 & 95,0 \\
\hline G3 & 22,0 & 482,5 & 405 & 331,1 & 68,6 & 11,0 & 10 & 6,1 & 90,7 & 16,4 & 15 & 7,7 & 91,7 \\
\hline G4 & 1,1 & 545,1 & 478 & 311,6 & 57,2 & 21,0 & 21 & 8,5 & 100,0 & 32,2 & 32 & 11,2 & 99,4 \\
\hline Gn1 & 1,1 & 370,5 & 446 & 161,5 & 43,6 & 2,6 & 2 & 2,4 & 75,5 & 4,1 & 3 & 3,0 & 73,8 \\
\hline Gn2 & 1,6 & 469,1 & 480 & 209,1 & 44,6 & 5,0 & 4 & 3,5 & 80,4 & 7,4 & 7 & 4,1 & 94,1 \\
\hline Gn3 & 2,8 & 468,3 & 355 & 395,2 & 84,4 & 9,7 & 9 & 5,7 & 92,5 & 14,4 & 13 & 7,2 & 90,5 \\
\hline Gn4 & 0,6 & 684,4 & 560 & 461,7 & 67,5 & 20,8 & 21 & 9,0 & 100,8 & 32,3 & 31 & 13,4 & 96,1 \\
\hline PC1 & 0,1 & 415,9 & 416 & 11,2 & 2,7 & 1,0 & 1 & 0,9 & 105,0 & 1,5 & 1 & 1,1 & 65,4 \\
\hline PC2 & 4,5 & 515,7 & 498 & 79,9 & 15,5 & 4,5 & 4 & 3,2 & 89,9 & 6,6 & 6 & 3,9 & 90,9 \\
\hline PC3 & 11,5 & 563,9 & 566 & 275,6 & 48,9 & 10,9 & 10 & 6,1 & 91,7 & 16,2 & 15 & 7,6 & 92,6 \\
\hline PC4 & 11,4 & 790,0 & 750 & 326,9 & 41,4 & 20,7 & 21 & 8,0 & 101,4 & 31,7 & 31 & 13,6 & 97,9 \\
\hline \multirow{2}{*}{$\begin{array}{l}\text { Tipos } \\
\text { lito }\end{array}$} & \multirow{2}{*}{$\begin{array}{c}\text { Superf. } \\
(\%)\end{array}$} & \multicolumn{4}{|c|}{ Altitud (A) } & \multicolumn{4}{|c|}{ Pendiente (S) } & \multicolumn{4}{|c|}{ Rugosidad (R) } \\
\hline & & $\mu$ & $\mathrm{ma}$ & sd & $\mathrm{CV}$ & $\mu$ & $\mathrm{ma}$ & sd & $\mathrm{CV}$ & $\mu$ & ma & sd & $\mathrm{CV}$ \\
\hline $\mathrm{D}$ & 8,5 & 364,7 & 403 & 240,2 & 65,9 & 3,2 & 2 & 4,3 & 132,9 & 5,2 & 3 & 6,2 & 119,8 \\
\hline E & 17,9 & 418,2 & 371 & 248,0 & 59,3 & 7,9 & 6 & 6,1 & 76,8 & 11,7 & 10 & 8,1 & 69,2 \\
\hline G & 40,4 & 474,2 & 446 & 295,0 & 62,2 & 8,6 & 7 & 6,5 & 75,0 & 12,8 & 11 & 8,7 & 67,7 \\
\hline Gn & 6,7 & 473,1 & 436 & 334,4 & 70,7 & 8,3 & 6 & 7,1 & 85,3 & 12,5 & 10 & 10,3 & 82,1 \\
\hline PC & 26,6 & 655,9 & 603 & 306,3 & 46,7 & 13,8 & 13 & 9,1 & 65,6 & 20,9 & 19 & 14,4 & 68,9 \\
\hline \multirow{2}{*}{$\begin{array}{l}\text { Tipos } \\
\text { topo }\end{array}$} & \multirow{2}{*}{$\begin{array}{c}\text { Superf. } \\
(\%)\end{array}$} & \multicolumn{4}{|c|}{ Altitud (A) } & \multicolumn{4}{|c|}{ Pendiente (S) } & \multicolumn{4}{|c|}{ Rugosidad (R) } \\
\hline & & $\mu$ & $\mathrm{ma}$ & $s d$ & $\mathrm{cV}$ & $\mu$ & $\mathrm{ma}$ & $\mathrm{sd}$ & $\mathrm{CV}$ & $\mu$ & ma & sd & $\mathrm{cV}$ \\
\hline 1 & 11,5 & 401,9 & 437 & 138,0 & 34,3 & 2,0 & 2 & 1,9 & 95,4 & 3,1 & 3 & 2,4 & 77,3 \\
\hline 2 & 30,9 & 425,8 & 432 & 175,3 & 41,2 & 4,9 & 4 & 3,5 & 72,1 & 7,4 & 7 & 4,3 & 58,3 \\
\hline 3 & 43,8 & 483,8 & 441 & 274,2 & 56,7 & 10,0 & 9 & 5,9 & 59,6 & 14,8 & 14 & 7,5 & 50,4 \\
\hline 4 & 13,9 & 682,3 & 622 & 335,6 & 49,2 & 20,3 & 21 & 8,3 & 40,7 & 31,1 & 31 & 11,8 & 37,9 \\
\hline
\end{tabular}


abarca, en sectores de escaso desarrollo espacial, los valores de celdas vecinas, por ejemplo, en tramos de ladera con fuertes pendientes y congostos valles, donde la pendiente del fondo de valle se sobredimensionará respecto a su valor real (Horacio y Ollero, 2011).

\subsection{Características de las variables de contraste}

El conjunto de la cuenca del río Cabe comprende 10 ULT con diferente porcentaje de ocupación: D1 (25,3\%), D2 (5,7\%), E4 (<1\%), G1 (4,9\%), G2 (10,6\%), G4 (0,7\%), Gn3 $(<1 \%)$, PC2 $(3,4 \%)$, PC3 $(35,5 \%)$ y PC4 $(7,9 \%)$. Si atendemos únicamente al cauce del río Cabe, este transita, en distinta proporción, por 7 ULT: D1 (40,2\%), D2 (5,5\%), G2 (14,5\%), G4 (5,0\%), Gn3 (2,9\%), PC3 (7,0\%) y PC4 $(25,0 \%)$. Respecto a los 23 puntos de muestreo trabajados en campo (Figura 3), el $39 \%$ se sitúa en la ULT D1, un 26\% en PC4, 13\% en Gn3, 13\% en G2 y 9\% en G4. La escala a la que se desarrolló la cartografía no refleja, al nivel de la cuenca del Cabe, toda la variedad de ULT, por lo que los últimos valores porcentuales, referidos a los puntos de muestreo, no se ajustan exactamente a la cartografía generada para Galicia (Figura 3). Este retoque manual, pero ajustado a la escala de la cuenca y por tanto más próximo a la realidad, se corresponde con los puntos Ca14, Ca15 y Ca17, que fueron ubicados en la ULT Gn3, en vez de la D1 que señala el mapa (Figura 3).

Tabla 2. Coeficiente de correlación de Spearman ( $\rho$ ) entre las variables de contraste, donde, el gris claro señala una "buena correlación" ( $\rho=0,6-0,8)$, gris oscuro una "muy buena correlación" ( $\rho=0,8-1)$ y "-" sin significancia estadística $(0,05)$

\begin{tabular}{|l|c|c|c|c|c|}
\hline & $S$ & $R h$ & $a / p$ & $D_{50}$ & $P$ \\
\hline $\mathrm{S}$ & 1 & - & $-0,79$ & 0,66 & 0,91 \\
$\mathrm{Rh}$ & - & 1 & 0,28 & - & - \\
$\mathrm{a} / \mathrm{p}$ & $-0,79$ & - & 1 & - & $-0,68$ \\
$\mathrm{D}_{50}$ & 0,66 & - & - & 1 & 0,63 \\
$\mathrm{P}$ & 0,91 & - & $-0,68$ & 0,63 & 1 \\
\hline
\end{tabular}

La correlación entre las variables de contraste muestra situaciones muy diferentes según el par analizado (Tabla 2). Las correlaciones son "buenas" o "muy buenas" ( $\sigma$ : $\pm 0,6$ - \pm 1 ), a pesar de que el número de casos sin significancia estadística es elevado. La pendiente está muy bien correlacionada con todos los factores -a excepción del radio hidráulico (sin significancia)-, lo que demuestra que esta variable posee una fuerte carga explicativa sobre las demás (véase Figura 4), en especial con la potencia específica 
$(0,91)$. Por el contrario, el radio hidráulico no presenta significancia estadística con ninguna variable. La ratio a/p se correlaciona inversamente con pendiente y potencia ($0,79,-0,68$, respectivamente). El $\mathrm{D}_{50}$ del sedimento tiene una intensidad de correlación con las otras variables similar a la que presenta la ratio $\mathrm{a} / \mathrm{p}$, aunque con signo positivo $(0,66,0,63$, respectivamente).

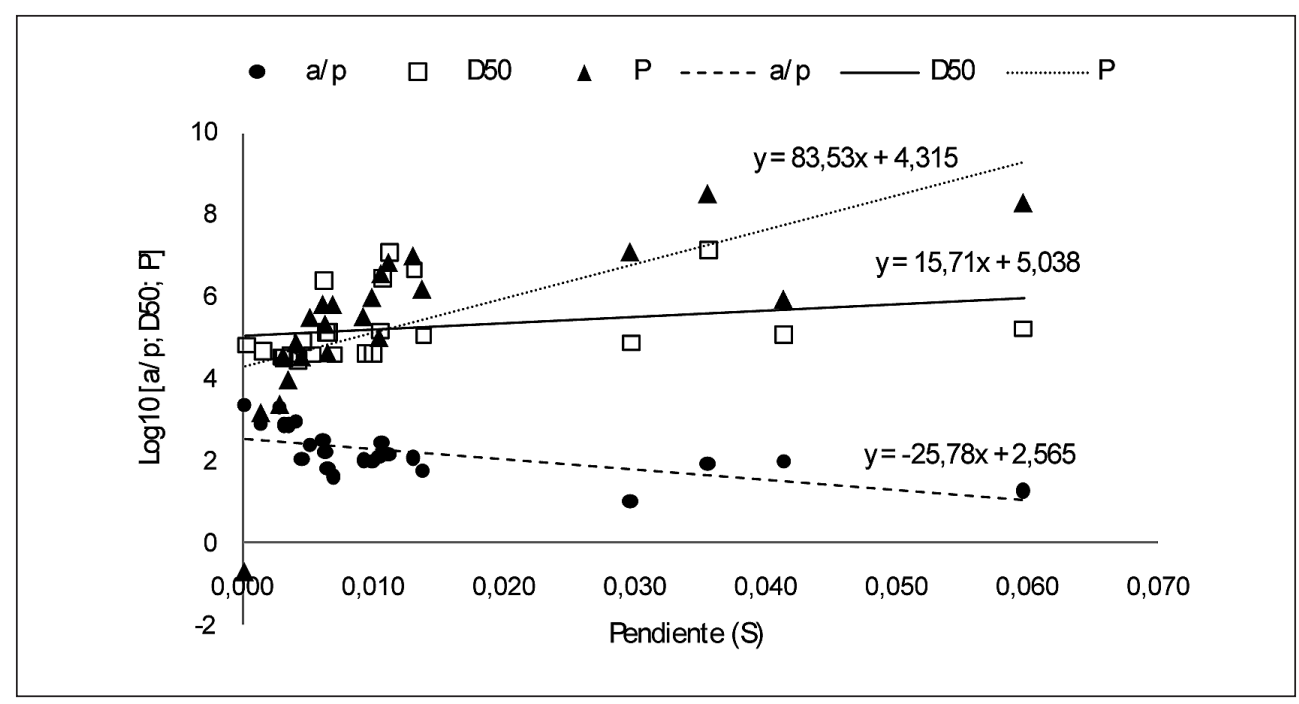

Figura 4. Relación de la pendiente con la potencia específica, la ratio a/p y la mediana del tamaño del sedimento $\left(D_{50}\right)$.

La pendiente predice el 83\% del comportamiento de la potencia, un 63\% de la ratio a/p y un $43 \%$ del $\mathrm{D}_{50}$. En el caso de la potencia específica, la pendiente forma parte de su ecuación, siendo además uno de los parámetros que más pondera y es responsable de dotar de energía al sistema. El coeficiente de determinación para la ratio a/p $(0,63)$ y, especialmente, para el $\mathrm{D}_{50}(0,43)$, está señalando que el valor predictivo de la pendiente es insuficiente y existen otros factores influyentes.

La variedad de correlaciones es sintomática de que las variables manejadas explican aspectos diferentes y con cierta independencia. Por tanto, contienen una carga explicativa diversa, lo que resulta de gran utilidad para contrastar y justificar las ULT como unidades internamente homogéneas y claramente diferenciables de las restantes.

En la Tabla 3 se recogen los datos de las 5 variables de contraste empleadas en los 23 puntos de muestreo repartidos a lo largo del río Cabe. Estos datos se han sometido a un análisis exploratorio que reflejase las características de cada variable (Tabla 4). El 
Tabla 3. Datos de las variables de contraste en cada punto de muestreo del río Cabe

\begin{tabular}{|l|l|l|l|r|r|r|}
\hline Id & ULT & $S$ & $R h$ & $a / p$ & \multicolumn{1}{c|}{$D_{50}$} & \multicolumn{1}{c|}{$P$} \\
\hline Ca02 & PC4 & 0,0414 & 0,38 & 7,23 & 161,65 & 382,27 \\
Ca03 & PC4 & 0,0598 & 1,05 & 3,55 & 187,91 & 4008,67 \\
Ca04 & PC4 & 0,0297 & 0,72 & 2,78 & 133,45 & 1207,93 \\
Ca06 & PC4 & 0,0138 & 1,09 & 5,70 & 156,05 & 487,75 \\
Ca08 & PC4 & 0,0069 & 1,55 & 5,01 & 100,22 & 335,38 \\
Ca09 & PC4 & 0,0065 & 0,91 & 6,17 & 170,76 & 103,46 \\
Ca10 & D1 & 0,0105 & 0,72 & 8,02 & 177,22 & 150,20 \\
Ca11 & D1 & 0,0092 & 1,06 & 7,41 & 101,75 & 249,54 \\
Ca12 & D1 & 0,0045 & 1,10 & 7,76 & 134,91 & 94,09 \\
Ca14 & Gn3 & 0,0063 & 1,50 & 9,06 & 169,88 & 207,79 \\
Ca15 & Gn3 & 0,0052 & 1,82 & 10,91 & 99,57 & 244,65 \\
Ca17 & Gn3 & 0,0099 & 1,79 & 7,22 & 102,79 & 399,79 \\
Ca18 & D1 & 0,0029 & 0,82 & 26,77 & 94,05 & 29,58 \\
Ca19 & D1 & 0,0035 & 1,51 & 17,59 & 99,26 & 53,73 \\
Ca20 & D1 & 0,0031 & 1,67 & 17,32 & 90,94 & 95,97 \\
Ca22 & D1 & 0,0014 & 1,54 & 17,88 & 107,37 & 24,09 \\
Ca23 & D1 & 0,0041 & 1,65 & 19,08 & 85,36 & 132,39 \\
Ca24 & D1 & 0,0002 & 1,29 & 27,98 & 124,38 & 0,50 \\
Ca25 & G2 & 0,0112 & 2,25 & 8,46 & 1208,68 & 932,99 \\
Ca26 & G2 & 0,0131 & 2,11 & 7,82 & 793,40 & 1095,59 \\
Ca27 & G2 & 0,0062 & 2,13 & 12,17 & 618,26 & 337,65 \\
Ca28 & G4 & 0,0106 & 2,16 & 11,39 & 650,09 & 710,08 \\
Ca29 & G4 & 0,0356 & 1,92 & 6,92 & 1294,44 & 4981,7 \\
\hline
\end{tabular}

valor más homogéneo en lo que a variabilidad se refiere es claramente el radio hidráulico. En el lado opuesto está la potencia específica, con un coeficiente de variación elevado $(1,8)$.

Los valores atípicos y, en especial, los extremos, pueden resultar de sumo interés en estudios de discriminación de unidades (Pardo y Ruiz, 2005), a diferencia de lo que sucede en trabajos de caracterización, en donde un valor anómalo desvirtúa el carácter general. Los valores extremos de la pendiente (Tabla 4) se sitúan en tramos de cabecera, salvo Ca29, que representa el cierre de cuenca. Si atendemos al tipo de unidad topográfica sobre la que se asientan estos valores extremos, todos ellos se enmarcan en el 
nivel topográfico 4 (ver Tabla 1). Más llamativos, y también esclarecedores, son los valores extremos de $\mathrm{D}_{50}$, puesto que se corresponden con las ULT G2 y G4. Es decir, el análisis exploratorio está indicando como la variable $\mathrm{D}_{50}$ sirve de elemento individualizador de ULT. La potencia contiene dos datos extremos (Tabla 4), Ca03 y Ca29, presentes también en la pendiente. Estos datos extremos de la pendiente y la potencia, además de estar altamente correlacionados $(0,91)$, se ubican en el mismo tipo de unidad topográfica (nivel 4), pero no litológica. Por el contrario, los datos extremos de la granulometría $\left(\mathrm{D}_{50}\right)$, pertenecen al grupo litológico de los granitoides, pero a diferentes zonas topográficas. Esto pone de manifiesto que hay ciertas variables que guardan una mayor relación con las unidades topográficas (pendiente y potencia), mientras que otras lo hacen con los dominios litológicos $\left(D_{50}\right)$. Por su parte, la geometría hidráulica, representada por el radio hidráulico y la ratio a/p, es dependiente o, si se prefiere, reflejo, de las condiciones topográficas, litológicas y de régimen (hidrológico y sedimentológico) del entorno. Los factores explicativos del perímetro y área mojada de un cauce (Rh) provienen de la suma de numerosos factores globales y locales, a los que el río se adapta y modela para dar salida al caudal líquido y sólido (Charlton, 2007; Malavoi y Bravard, 2010).

Tabla 4. Análisis exploratorio de las variables de contraste

\begin{tabular}{|l|c|c|c|c|c|c|c|c|c|c|c|}
\hline Variable & $N$ & Rango & Mín & Máx & $m a$ & & $s d$ & Varianza & cv & atípico & extremo \\
\hline $\mathrm{S}$ & 23 & 0,06 & 0,0002 & 0,06 & 0,0 & 0,013 & 0,015 & 0,0002 & 1,1 & 4 & $2,3,29$ \\
$\mathrm{Rh}$ & 23 & 1,9 & 0,38 & 2,25 & 1,5 & 1,4 & 0,5 & 0,3 & 0,4 & & \\
$\mathrm{a} / \mathrm{p}$ & 23 & 25,2 & 2,8 & 28,0 & 8,0 & 11,1 & 6,9 & 47,4 & 0,6 & 18,24 & \\
$\mathrm{D}_{50}$ & 23 & 1209,1 & 85,4 & 1294,4 & 134,9 & 298,4 & 359,1 & 128940,5 & 1,2 & & 25,26, \\
& & & & & & & & & & & $\begin{array}{c}27,28 \\
29\end{array}$ \\
$\mathrm{P}$ & 23 & 4981,2 & 0,5 & 4981,7 & 249,5 & 707,2 & 1250,9 & 1564836,9 & 1,8 & & 3,29 \\
\hline
\end{tabular}

\subsection{Características de las ULT según las variables de contraste}

Se ha evaluado mediante un análisis ANOVA si existen diferencias entre las distintas ULT de la cuenca del río Cabe (Figura 3). Según el test de Shapiro-Wilk, tan solo los valores de potencia de la ULT PC4 no se ajustan a una distribución normal (p-valor: 0,006), lo que ha requerido de un proceso de normalización mediante un logaritmo neperiano (p-valor: 0,851). Por otra parte, al contar la ULT G4 únicamente con dos puntos de muestreo, no se puede determinar su normalidad. Si bien, considerando la unidad litológica granitoide (G2 + G4), los datos se ajustan a la normalidad, a excepción de la pendiente y potencia, debido al efecto perturbador que introduce el extremo Ca29 (Tabla 4). 
La homogeneidad de varianzas de las variables entre las ULT se contrastó por medio del test de Levene. El radio hidráulico y la ratio a/p cumplen las condiciones de homocedasticidad ( $\mathrm{p}$-valor, respectivamente: 0,184, 0,050), pero no las restantes variables. Para estos casos las varianzas se homogeneizaron mediante una transformación logarítmica, cumpliendo, además, el supuesto de normalidad. En el análisis de pendiente y potencia se ha descartado el punto de muestreo Ca24, puesto que la obtención de la pendiente en campo en este punto es de dudosa calidad, lo que podría distorsionar los resultados finales de esta variable y, por ende, de la potencia específica.

El análisis ANOVA ha demostrado que existen disimilitudes entre las ULT. Este mismo análisis, aplicado sobre las unidades topográficas y litológicas por separado, arroja idénticas conclusiones. En la Tabla 5 se muestran las comparaciones múltiples post hoc realizadas mediante el estadístico de Bonferroni, comprobándose cómo las variables tienen un comportamiento diferenciado según la ULT. La potencia no es significativamente diferente entre PC4, Gn3, G2 y G4, pero sí aparece desmarcada en D1, que únicamente guarda parecido con Gn3. En el caso de la pendiente, tan solo se dan diferencias significativas entre PC4 y D1. El $\mathrm{D}_{50}$ establece dos grupos bien diferenciados, el de los granitoides (G2 y G4) y el resto, y, en efecto, así lo reflejan también los subgrupos homogéneos de la prueba de Tukey. La ratio a/p presenta un comportamiento idéntico a la pendiente $(\rho:-0,79)$. Su valor oscila en un rango parecido para Gn3, G2 y G4, pero decae ostensiblemente en PC4 (mayor encajamiento) y se acentúa en D1, donde dominan más los procesos expansivos que incisivos. Los resultados del radio hidráulico denotan una gran dependencia de la cuenca vertiente ( $\mathrm{r}$ de Pearson=0,77), de modo que cada ULT se comporta como un grupo propio (porque propia lo es también su cuenca de drenaje), salvo G2 y G4, cuyas medias se encuentran en un nivel parejo. La prueba de Tukey establece para el radio hidráulico tres grupos correlativos desde cabecera hasta desembocadura: PC4 y D1; Gn3 y D1; Gn3, G2 y G4.

Las comparaciones múltiples por grupos litológicos (Tabla 5) siguen, como cabría esperar, la pauta descrita para las ULT. Las manchas litológicas constituyen unidades bastante compactas espacialmente, sin una elevada atomización. En el caso de los grupos topográficos (Tabla 5), los resultados son mucho más cambiantes que los dados en los tipos litológicos. Los primeros siguen una secuencia desde cabecera hasta desembocadura, sin repetirse unidades en el transcurrir del río; sin embargo, no sucede así con la zonificación topográfica. Esto hace que, por ejemplo, el nivel topográfico 1 (ver Figura 3) aparezca en cabecera y desembocadura, con unas dimensiones del cauce o de la granulometría del sedimento claramente diferentes. El $\mathrm{D}_{50}$ de los tipos topográficos 1 y 4 es significativamente diferente debido a que la tipología 4 engloba las ULT PC4 y G4, mientras que el tipo 1 se corresponde íntegramente con la ULT D1 (ver Tabla 3). Estas diferencias explican, por ejemplo, porqué la mediana de la granulometría en la unidad topográfica 1 (por tanto también en D1) se mantiene baja, pero alta en la unidad 4, por tanto también en PC4, debido en este caso al aporte de tamaño que supone el sector granitoide del sur de la cuenca (G2, G4). 


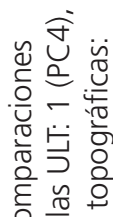

응 ฯ 䒕 市

준 凶.든 증

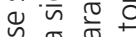

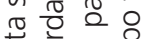

学离宁这

ब

嵌

$\cdot \dot{0} \mathrm{O}$

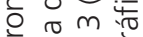

屯 है ठํㅇ

ㅇํ응 응

응 으을

요욜

氜

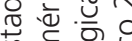

ขै

ब

도는 응

융

$\tilde{u}$

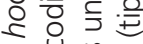

藏范

$8 \rightarrow \frac{0}{=}$

啇望。

을

产

$\varepsilon .{ }^{2}$ ㄴ

थ

은 능응

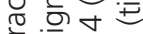

证

ह ํㄷㄷ

¿

. ट $\mathrm{m}$

을

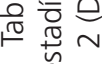

\begin{tabular}{|c|c|c|c|c|c|c|c|c|c|c|c|c|c|c|c|}
\hline \multirow{5}{*}{ 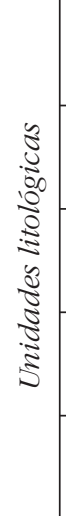 } & 2 & 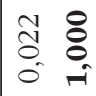 & $\stackrel{8}{8}$ & & & \begin{tabular}{l}
1 \\
\multirow{2}{*}{} \\
0
\end{tabular} & \& & & \begin{tabular}{l}
$\hat{1}$ \\
\multirow{2}{*}{} \\
0
\end{tabular} & ஜ & & & $\begin{array}{l}0 \\
8 \\
0\end{array}$ & $\stackrel{8}{8}$ & \\
\hline & $a^{\text {in }}$ & 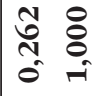 & $\begin{array}{l}8 \\
8 \\
0\end{array}$ & & $\begin{array}{l}\text { ปै } \\
\text { กิ } \\
0\end{array}$ & $\stackrel{8}{8}$ & $\begin{array}{l}8 \\
8 \\
0\end{array}$ & \&̊ & $\stackrel{8}{\circ}$ & $\begin{array}{l}8 \\
\vdots \\
0\end{array}$ & & & $\begin{array}{l}8 \\
8 \\
0 \\
0\end{array}$ & $\begin{array}{l}8 \\
8 \\
0\end{array}$ & \\
\hline & $\sum_{i}^{2}$ & $\begin{array}{ll}n & 8 \\
8 & 8 \\
0 & 0 \\
0 & -1\end{array}$ & $\overbrace{-1}$ & & & ֻै & $\frac{n}{2}$ & ס̊ & 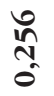 & $\stackrel{8}{8}$ & & & के & $\stackrel{8}{\circ}$ & \\
\hline & व्य & $\mid \begin{array}{ll}0 & 0 \\
0 & 0 \\
+ & 0 \\
0 & 0\end{array}$ & $\begin{array}{l}8 \\
8 \\
0\end{array}$ & & & हे & $\begin{array}{l}\overrightarrow{8} \\
0 \\
0\end{array}$ & & $\begin{array}{l}-1 \\
0 \\
\text { ñ } \\
0\end{array}$ & $\begin{array}{l}\text { in } \\
\text { ñ } \\
0\end{array}$ & & & $\begin{array}{l}5 \\
8 \\
0\end{array}$ & in & \\
\hline & $\infty$ & $\begin{array}{ll}0 & 0 \\
0 & \hat{n} \\
0 & 0\end{array}$ & $\stackrel{8}{8}$ & & & $\stackrel{8}{8}$ & $\begin{array}{l}\infty \\
\infty \\
0 \\
0\end{array}$ & ôे & @i & @ & & & $\begin{array}{l}\infty \\
0 \\
0 \\
0\end{array}$ & $\stackrel{8}{8}$ & \\
\hline \multirow{5}{*}{ 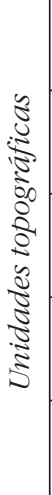 } & 2 & $\mid$\begin{tabular}{ll}
1 & \multirow{+}{*}{} \\
0 & 0 \\
0 & 0
\end{tabular} & $\begin{array}{l}8 \\
0\end{array}$ & & & \& & $\stackrel{8}{\circ}$ & & : & $\stackrel{8}{8}$ & & & $\stackrel{8}{-}$ & $\stackrel{8}{\circ}$ & \\
\hline & $a^{i n}$ & $\begin{array}{ll}8 & 8 \\
0 & -\end{array}$ & \$े & & & $\stackrel{1}{0}$ & $\begin{array}{l}0 \\
0 \\
0\end{array}$ & & $\begin{array}{l}0 \\
0 \\
0\end{array}$ & $\stackrel{8}{-}$ & & & $\begin{array}{l}\hat{0} \\
0\end{array}$ & $\stackrel{8}{-1}$ & \\
\hline & $\frac{\pi}{2}$ & 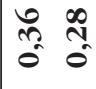 & $\begin{array}{l}8 \\
0\end{array}$ & & & $\stackrel{8}{-}$ & $\stackrel{8}{\circ}$ & & @i & ᄋ & & & $\stackrel{8}{\circ}$ & $\stackrel{8}{\circ}$ & \\
\hline & $\frac{\sqrt{x}}{x}$ & $\begin{array}{ll}+ & 0 \\
0 & \infty \\
0 & 0\end{array}$ & $\stackrel{8}{8}$ & & & $\stackrel{8}{8}$ & $\begin{array}{l}n \\
0 \\
0\end{array}$ & & $\stackrel{8}{8}$ & $\begin{array}{l}n \\
0\end{array}$ & & & $\begin{array}{l}n \\
0 \\
0\end{array}$ & $\frac{m}{0}$ & \\
\hline & $\infty$ & $\stackrel{1}{A}$ & $\begin{array}{l}8 \\
0\end{array}$ & & & $\stackrel{8}{\circ}$ & $\stackrel{8}{\circ}$ & & $\stackrel{-}{8}$ & $\hat{n}$ & & & $\stackrel{8}{8}$ & $\hat{n}$ & \\
\hline \multirow{7}{*}{$\stackrel{5}{S}$} & 2 & $\begin{array}{ll}+1 & 0 \\
0 & 0 \\
0 & 0 \\
0 & -1\end{array}$ & & & & $\frac{n}{2}$ & 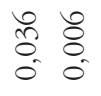 & 兽 & $\hat{n}$ & & है. & & 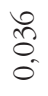 & $\overbrace{0} 8$ & $\begin{array}{llll}8 & 8 & \text { n } & 8 \\
0 & 0 & \text { nิ } & 0 \\
\text { - } & 0 & 0 & -1\end{array}$ \\
\hline & $a^{\text {in }}$ & \begin{tabular}{ll}
0 & 8 \\
\multirow{1}{*}{} & 8 \\
0 & 0 \\
0 & -1
\end{tabular} & & & & $\stackrel{8}{8}$ & $\begin{array}{ll}8 & 8 \\
8 & 8 \\
0 & 0\end{array}$ & $\underset{8}{8}$ & రి & $\begin{array}{l}8 \\
8 \\
0\end{array}$ & $\begin{array}{l}8 \\
8 \\
0\end{array}$ & 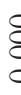 & $\begin{array}{l}8 \\
8 \\
0\end{array}$ & $\begin{array}{ll}8 \\
8 & 8 \\
0 & 0 \\
-1\end{array}$ & $\begin{array}{llll}8 & 8 & 8 & 8 \\
8 & 8 & 8 & 8 \\
0 & 0 & 0 & -\end{array}$ \\
\hline & $\frac{n}{\pi}$ & $\begin{array}{ll}1 & 0 \\
0 & 8 \\
0 & 0 \\
0 & -1\end{array}$ & $\stackrel{-}{8}$ & $\underset{8}{8}$ & & 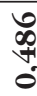 & $\begin{array}{ll}0 & n \\
\bar{\sigma} & \bar{\sigma} \\
0 & 0\end{array}$ & $\underset{8}{8}$ & $\begin{array}{l}\infty \\
\infty \\
+ \\
0 \\
0\end{array}$ & $\underset{8}{8}$ & $\stackrel{8}{8}$ & 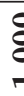 & $\begin{array}{l}0 \\
\text { ] } \\
0 \\
0\end{array}$ & 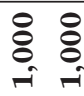 & 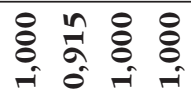 \\
\hline & व्य & $\begin{array}{ll}1 & 0 \\
\infty & 0 \\
\infty & 0 \\
0 & 0\end{array}$ & $\begin{array}{l}8 \\
8 \\
0\end{array}$ & & & $\begin{array}{l}\infty \\
1 n \\
0\end{array}$ & $\begin{array}{ll}0 & 1 \\
8 & 0 \\
0 & 0 \\
0 & 0\end{array}$ & $\begin{array}{l}0 \\
0 \\
0\end{array}$ & $\begin{array}{l}\infty \\
n \\
n \\
0\end{array}$ & $\begin{array}{l}\hat{\infty} \\
\hat{\sigma}\end{array}$ & $\stackrel{8}{\circ}$ & & $\begin{array}{l}8 \\
8 \\
0\end{array}$ & $\begin{array}{ll}1 & 8 \\
\infty & 8 \\
\sigma_{0} & 0 \\
0 & -1\end{array}$ & $\begin{array}{llll}0 & n & 0 & 0 \\
8 & 0 & 8 & 8 \\
0 & 0 & 0 & 0 \\
0 & 0 & -1 & -1\end{array}$ \\
\hline & $\infty$ & $\begin{array}{ll}0 & \hat{1} \\
0 & \hat{n} \\
0 & 0\end{array}$ & $\overbrace{0}$ & & & $\stackrel{8}{8}$ & 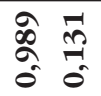 & in & $\stackrel{8}{8}$ & $\underset{8}{8}$ & $\underset{8}{8}$ & & $\begin{array}{l}\infty \\
\hat{\sigma}\end{array}$ & $\bigodot_{0} \underset{0}{0}$ & 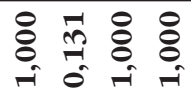 \\
\hline & & $r m$ & ঋ & & & $n$ & tin & & $v$ & $\psi$ & & & v & $n$ in & $r N m$ \\
\hline & & -1 & & & $N$ & & & $n$ & & & & 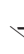 & & & in \\
\hline
\end{tabular}


Según los análisis comentados, se deduce que hay ciertos parámetros que son dependientes del tamaño de cuenca, por otros que no, sino más bien del tipo de ULT. Por ejemplo, variables como la anchura del cauce $(\rho=0,80)$ o el radio hidráulico $(\rho=0,77)$, están bien correlacionados con el tamaño de la cuenca vertiente, y no con el tipo de ULT. No obstante, estas sí se correlacionan bien con variables que no lo hacen con las dimensiones de cuenca, como la pendiente $(\rho=-0,32)$, la ratio a/p $(\rho=0,62)$, el $\mathrm{D}_{50}(\rho=$ $0,20)$ o la potencia $(\rho=-0,46)$. Del mismo modo, todos aquellos parámetros morfométricos referidos al tamaño y forma de la cuenca vertiente no son aplicables sobre las ULT.

\subsection{Clasificación de las variables de contraste}

En la Figura 5 se muestra el dendrograma resultante de aplicar sobre los puntos de muestreo una clasificación jerárquica aglomerativa según el algoritmo de Ward (Emery et al., 2003). Para el nivel 1 (distancia de 3,1) se produce la organización más acorde de los tramos con las ULT. En este nivel se observan cuatro agrupaciones bien definidas: i) el primer grupo se corresponde con las ULT G2 y G4; ii) el segundo con el sector central de la ULT D1, inmediatamente después de que el río atraviesa el último sector gneísico (Gn3), que es, precisamente, el punto a partir del cual se dan las condiciones de mayor aplanamiento en la depresión de Lemos (ver Figura 1); iii) el tercero lo conforman los sectores de cabecera, por tanto, dentro de PC4, y iv) un cuarto más variado que engloba el sector bajo de PC4 hasta Gn3 y, por consiguiente, incluye también varios puntos de D1. El nivel 2 (Figura 5) tan solo difiere del anterior en que incorpora, a una distancia de 4,3, el tramo Ca29 al grupo de granitoides.

La línea de distancia de corte adaptada a las ULT (Figura 5) individualiza 5 clusters. Los dos grupos de mayor similitud se cierran a 1,2 de distancia. El primero representa a la ULT Gn3 donde también se incorpora el punto Ca08 de la ULT PC4. El segundo grupo es un híbrido $(\mathrm{H})$ donde se llegan a juntar puntos de tres ULT diferentes. La principal peculiaridad de este cluster es que, a pesar de no encajarse en ninguna ULT concreta, está englobando casi de forma continua todos los puntos desde Ca06 (inicio del sector con menor accidentalidad de PC4) hasta Ca12 (D1), punto que precede a la ULT Gn3.

El siguiente nivel en formarse es el de D1 (distancia 2,4) y en él se enmarcan todos los puntos desde Ca18, punto posterior a la ULT Gn3, hasta Ca24, punto precedente a G2. Con una distancia de 3,1 se forma el sector de cabecera (PC4) y a 4,3 se cierra la ULT granitoide (G2 y G4); si bien, básicamente se constituye a 1,9, tan solo a falta de Ca29 (es esta observación la que eleva la distancia a 4,3).

Se ha comprobado también cómo, por variables, son pendiente y potencia las primeras en unificarse (Figura 5). Le siguen el $\mathrm{D}_{50} \mathrm{y}$ el radio hidráulico, a los que posteriormente se une la ratio a/p. La carga explicativa de la pendiente sobre la potencia justifica el primer cluster, pero en el caso de $\mathrm{D}_{50}$ con radio hidráulico consideramos que su agrupación por semejanza se debe al hecho de que, longitudinalmente, esto es, en 


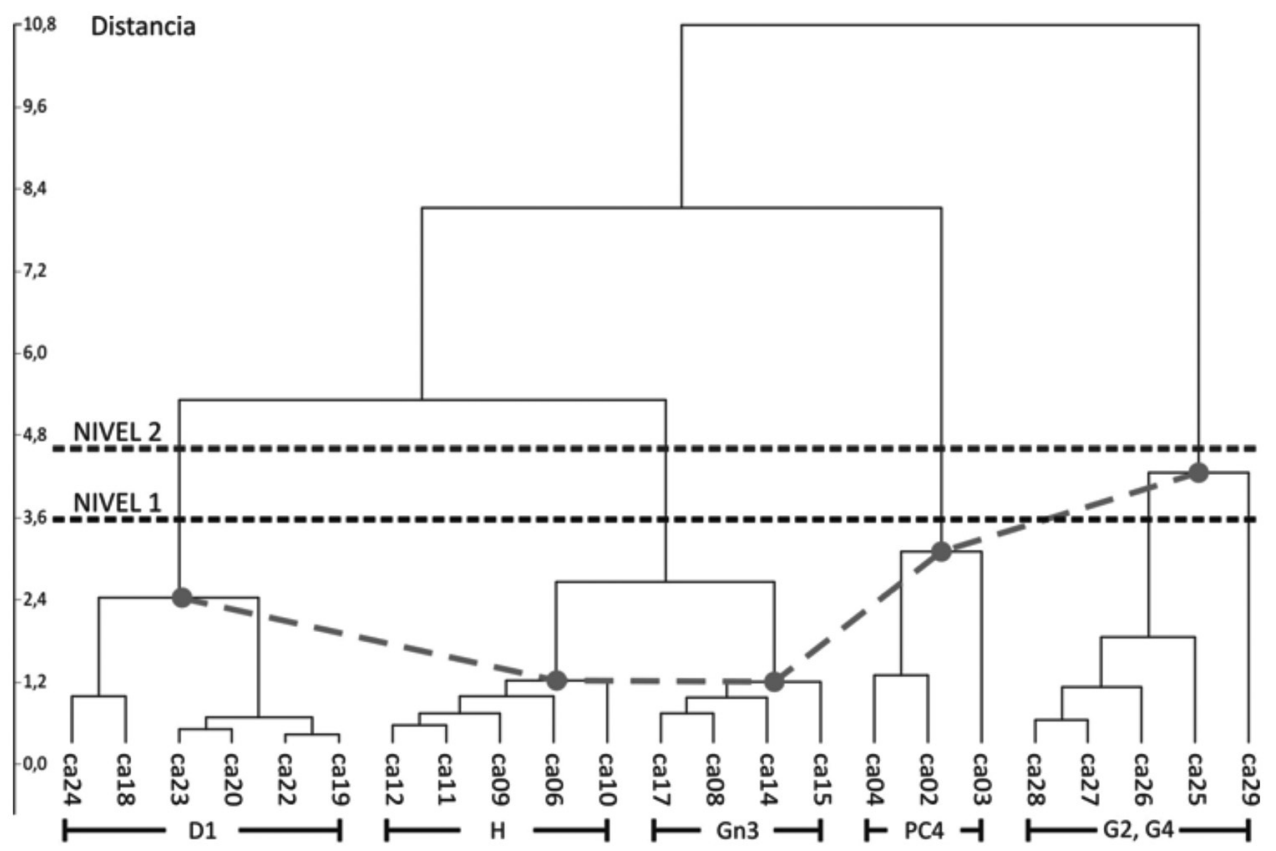

Figura 5. Dendrograma de los 23 puntos de muestreo del río Cabe según las variables de contraste (H: grupo híbrido).

dirección cabecera-desembocadura, los valores de ambas variables son crecientes. Este comportamiento ascendente lo provoca, en el caso del $\mathrm{D}_{50}$, el grupo granitoide de la zona baja de cuenca, mientras que en el radio hidráulico, como ya ha sido comentado, su incremento está altamente correlacionado con la cuenca vertiente.

De las agrupaciones hechas sobre el mapa factorial (Figura 6) para las dos primeras componentes principales (más del 85\% de la varianza) se desprenden unas conclusiones similares al análisis del dendrograma (Figura 5). Las ULT de granitoides (G2, G4) y D1 aparecen bastante compactas, lo mismo que PC4, aunque sin todos sus componentes. La nube de la ULT Gn3 almacena, a parte de los componentes de esa ULT, el punto Ca02 (PC4) y Ca10, Ca11 y Ca12 de la ULT D1. Tanto en el análisis cluster como en el factorial, se observa cómo las ULT granitoides y el sector de D1, a partir de Ca18 inclusive, guardan unas características comunes. Las mayores dificultades para agrupar aparecen entre Gn3 y el final de PC4; es decir, los contrastes entre estos puntos de muestreo no son altamente diferenciables. Según el gráfico factorial (Figura 6), Ca03 y Ca29 se comportan como observaciones con alguna particularidad que las aleja de la tónica general y que, para ambos casos, es provocada por el valor tan elevado de potencia específica (ver también Tabla 4). 

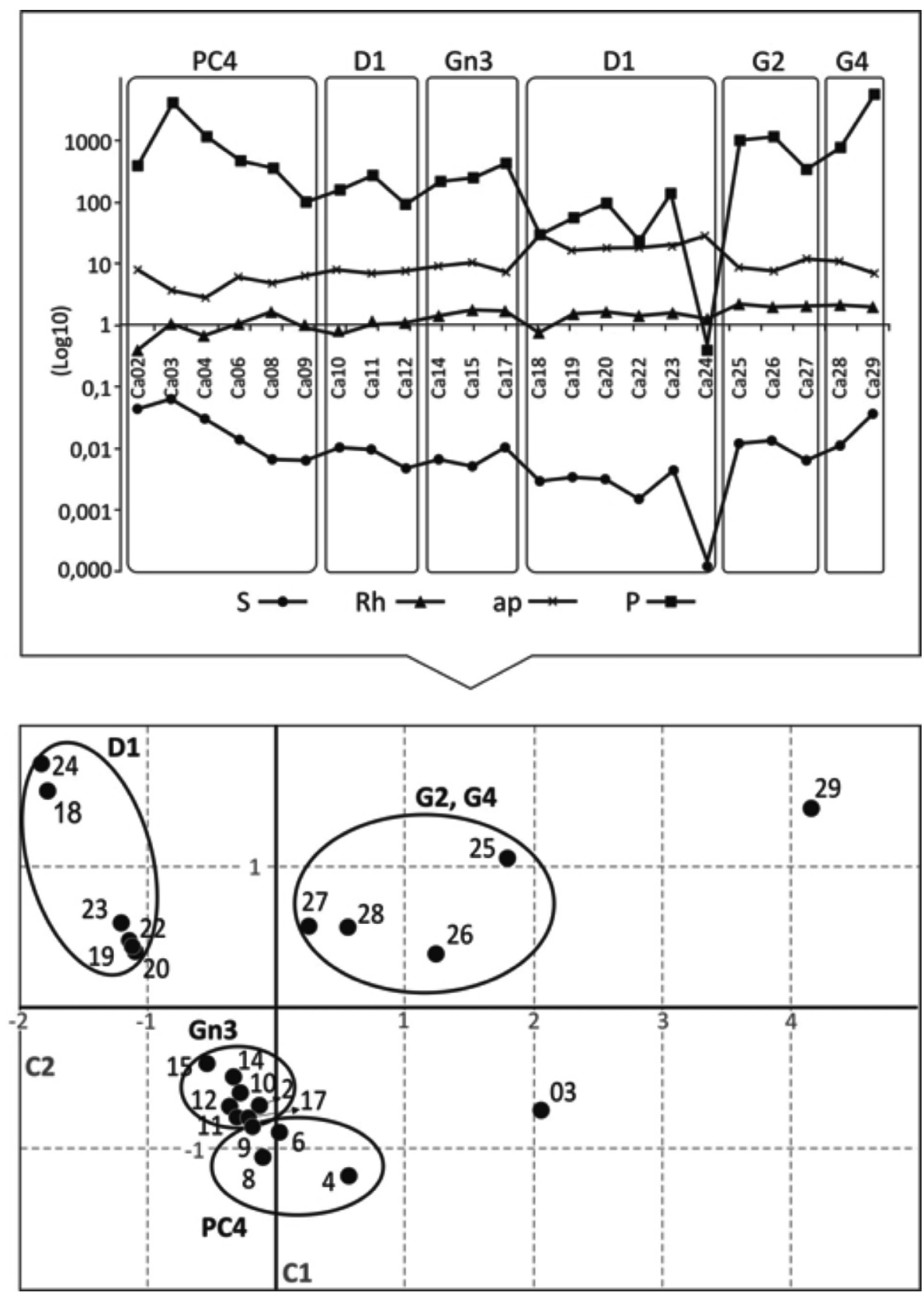

Figura 6. Relación de las 5 variables de contraste según cada punto de muestreo del río Cabe y agrupación factorial de los puntos de muestreo. 


\section{Discusión}

La interpretación que se hace de las ULT debe ponerse en relación con el sistema de caracterización jerárquica de los ríos propuesto por la DMA y recogido por González del Tánago y García de Jalón (2007). Los niveles organizativos de menor escala, ecorregiones y cuencas vertientes, deben servir para separar y explicar tipos de ríos. La guía de González del Tánago y García de Jalón (2007) señala que la ecorregión se emplea para "la diferenciación de las regiones con distintas condiciones hidrológicas y ecológicas" (p. 52), si bien, dentro de una misma ecorregión, "pueden existir ríos muy distintos, cuyas diferencias se pueden explicar en un segundo nivel de caracterización por las características de sus respectivas cuencas vertientes, definidas en este caso por su tamaño y por su geología" (p. 55). La Real Academia Española de la Lengua define el término caracterizar como la determinación de los atributos peculiares de algo, de modo que claramente se distinga de los demás. En este sentido, en efecto, una ecorregión es capaz de englobar comportamientos fluviales muy diferentes (Hawkins y Norris, 2000; CEDEX, 2004), pero de un modo muy sucinto y nunca con un grado de detalle tal como para explicar procesos y formas fluviales. Se considera que este primer nivel de regionalización es idóneo para contextualizar (Omernik y Bailey, 1997).

Con el segundo nivel de la organización jerárquica, la cuenca vertiente, se estructura el territorio hidrológicamente y/o como unidad de gestión (Chorley, 1969; Lotspecich, 1980). Su caracterización por tamaños es predictiva para la magnitud de las aportaciones e, incluso, se podría esbozar el tipo de trazado en planta de los ríos (González del Tánago y García de Jalón, 2007). En cuanto a la caracterización por criterios geológicos, la propuesta de la DMA de tres tipos (calcáreo, silíceo, orgánico) es incompleta e inadaptada a la diversidad de geologías existentes en los países miembros (Ollero et al., 2003; Ollero et al., 2010). González del Tánago y García de Jalón (2007) proponen el mapa de las litologías presentes en la Península Ibérica ilustrado en Gutiérrez-Elorza (1994), sintetizándose también en tres las litologías dominantes: silícea, caliza y arcillosa (Hernández-Pacheco, 1934).

La caracterización de la DMA engloba cuencas muy distintas dentro de un mismo rango. Valga de ejemplo la comparativa entre la cuenca del río aquí estudiado, Cabe, con la cuenca limítrofe por el este, la del río Lor, de $369 \mathrm{~km}^{2}$ y $95 \%$ perteneciente a la ULT PC4 (ver su localización en Figura 3). Según la DMA (adaptada parcialmente para España por la Guía Metodológica de Restauración de Ríos), ambas cuencas pertenecen al mismo tipo de ecorregión, de tipo de vegetación de ribera y de tamaño de cuenca. No obstante, las diferencias geomorfológicas, hidrológicas y, en consecuencia, de hábitats son significativas para el conjunto de cada cuenca. La cuenca del Lor es muy uniforme litológicamente (pizarro-cuarcítica, con algunas bandas calizas), de relieve con fuertes pendientes y con un caudal específico alto para el conjunto de Galicia y el más elevado de la provincia de Lugo con 38,5 1/s/ $/ \mathrm{km}^{2}$ (Carrión, 2001). La cuenca del Cabe engloba diferentes dominios litológicos (pizarras y cuarcitas, grupo de granitoides, de- 
pósitos o gneis), con una tipología de relieve también muy heterogénea y el caudal relativo más bajo de la provincia de Lugo $\left(12,9 \mathrm{l} / \mathrm{s} / \mathrm{km}^{2}\right)$. En la comparación de estas cuencas se 'rompen' algunos de los supuestos caracterizadores establecidos por la DMA. Por ejemplo, la relación área-caudal medio (Strahler, 1964) es mayor en el Lor que en el Cabe, a pesar de que esta última cuenca tiene el doble de superficie que la primera. El tipo de trazado en planta de ambos ríos difiere, aun en igualdad de superficie de cuencas vertientes, a lo largo de todo su recorrido. El aumento del caudal y de la cuenca vertiente hacia aguas abajo no supone para la cuenca del Cabe un descenso de la pendiente longitudinal del cauce, sino que, por el contrario, esta es en los tramos bajos mucho más elevada incluso que muchos sectores de cabecera. El incremento del caudal y cuenca vertiente no se relaciona positivamente con un incremento de la sinuosidad del río Cabe, aunque sí se observa una mayor meandrización del río Lor en el sector bajo de cuenca, pero posiblemente más atribuible a hechos estructurales que hidrológicos (Martín-Serrano, 1994; De Vicente et al., 2007).

Considerar la cuenca vertiente como segundo nivel de caracterización, en función de su tamaño y el grado de detalle geológico al que propone la DMA, no informa necesariamente sobre tramos fluviales con características similares que, a la postre, influyan y diferencien los tipos de hábitats y sus comunidades biológicas asociadas. La incorporación de la cartografía ULT sobre la cuenca vertiente supone su zonificación en áreas con unos procesos y dinámicas geomórficas similares, a los cuales se les atribuye un hábitat concreto. De este modo, se propicia que las comunidades biológicas con propiedades similares tengan más en común dentro de una ULT que atraviesa varias cuencas que dentro de varias ULT que conformen una sola cuenca. De la comparación de ULT para las cuencas de los ríos Cabe y Lor (Figura 3), se obtiene que la primera presenta un comportamiento más heterogéneo, atravesando el río diversas ULT. En cambio, el Lor transita por una sola ULT, lo que se puede interpretar como que su comportamiento geomorfológico y tipo de hábitats fluviales es bastante similar en cualquier punto del río.

La información aportada por el modelo de caracterización de la DMA para los dos primeros niveles escalares en los puntos Ca03, Ca15, Ca20 y Ca26 (un punto de muestreo por cada tipo de ULT), es de unos sectores situados en la Provincia Atlántica Europea (subprovincia Cántabro-Atlántica), en la región de Galicia de vegetación de ribera, con una litología silícea, o silícea-arcillosa, una altitud media y un tamaño de cuenca, respectivamente $\left(\mathrm{km}^{2}\right)$, de: 2,0; 155,3; 461,3 y 688,0. Toda esta información orienta sobre el tipo de hábitat, pero el grado de incertidumbre es muy elevado. La inclusión del tipo de ULT para cada uno de estos puntos (Tabla 1 y Tabla 3) supone reducir de forma notable la incertidumbre de aproximación para un tipo de hábitat. La ULT informa de qué tipo de granulometría domina en el hábitat, el nivel de encajamiento del cauce y, especialmente, la capacidad erosiva del río (potencia específica). Las posibilidades de despliegue energético del río son uno de los factores que más repercute en el tipo de hábitat (CEN, 2002) y, de un modo especial, en la vegetación de ribera (Hupp y Osterkamp, 1996). 
En la Figura 7 se incorporan las ULT al sistema de caracterización jerárquica de hábitats fluviales de la DMA, siendo su nivel escalar el mismo que la cuenca vertiente. Las ULT contienen toda la carga explicativa de la estructura (litología y tectónica), mientras que la cuenca vertiente se caracteriza únicamente por su tamaño. En cualquier caso, ambos factores son complementarios y nunca excluyentes: la ULT refuerza el análisis de la influencia geomorfológica en los ecosistemas (Montgomery, 1999; Ibisate et al., 2011); en cambio, la cuenca, explica los procesos ligados a su tamaño y, además, delimita un sector lógico para evaluar los impactos geomorfológicos sobre los procesos ecológicos, de ahí que proporcione un marco de gestión y análisis ideal en la dirección de recursos naturales (Newson, 2002).

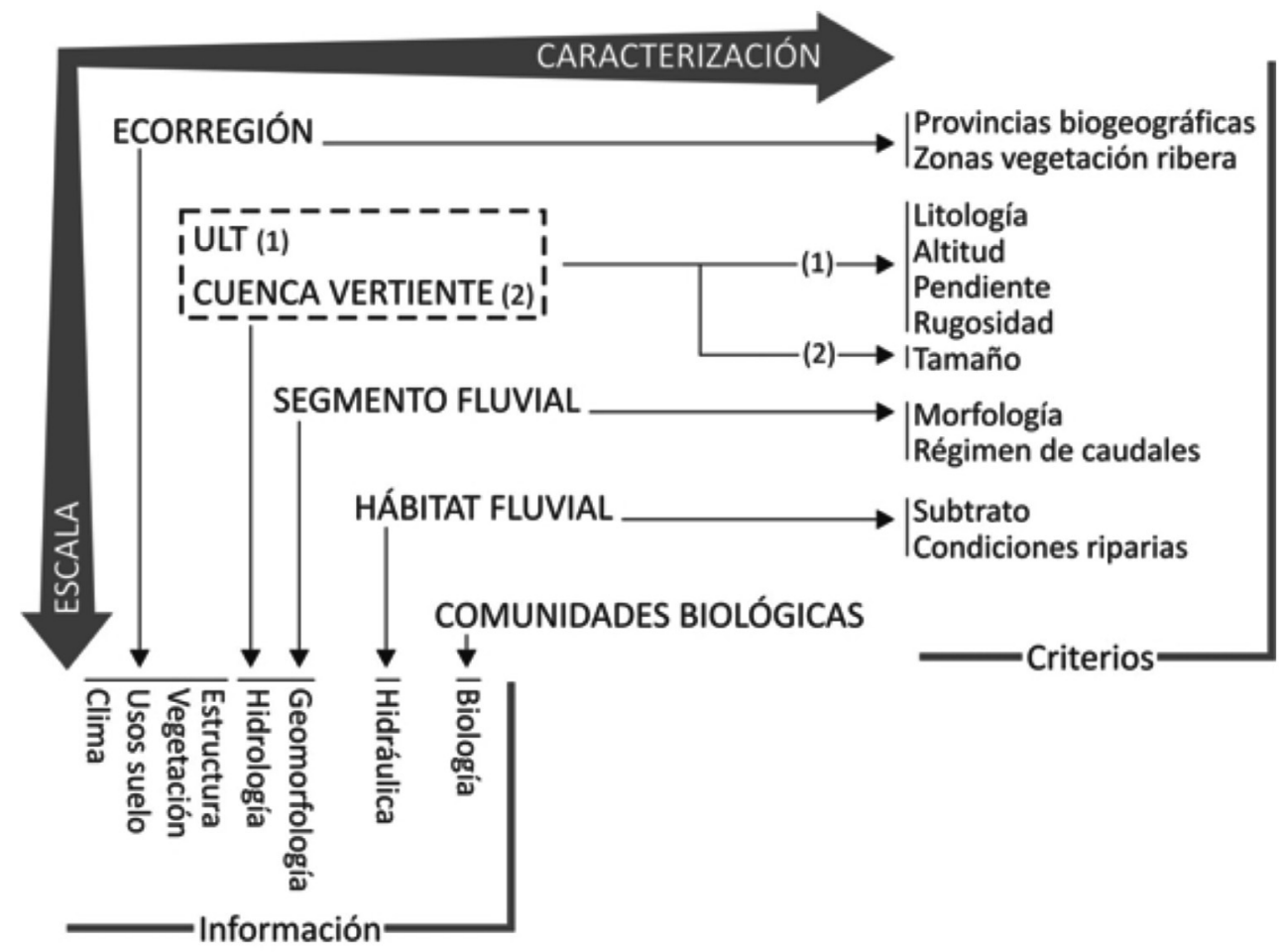

Figura 7. Esquema de caracterización jerárquica de los ríos incluyendo las ULT. La figura se organiza según la escala y grado de caracterización hasta el nivel de comunidad biológica.

El nuevo esquema que se dibuja con la incorporación de la ULT (Figura 7) al modelo de caracterización fluvial, destaca por las siguientes razones: i) dividir la litología en tres grandes grupos para toda la Península Ibérica no resulta muy aclarativo, sino más bien intuitivo de algunas características; ii) la carga explicativa de la litología se 
reduce interpretándola de forma aislada o con otras variables escasamente correlacionadas (tamaño de cuenca); iii) la litología gana en carga explicativa con la variable topográfica, con la que está mucho más correlacionada y, en conjunto, dan forma al hecho estructural de un territorio. En una región como Galicia, considerar y ponderar la componente estructural es fundamental (Martín-Serrano, 1989, 1991; Pérez-Alberti, 1982, 1991, 1993; Vidal-Romaní, 1996). Son precisamente las características estructurales las responsables de que, en numerosos casos, y parafraseando a W.M. Davis, los ríos 'nazcan viejos' y 'mueran jóvenes' (Pérez-Alberti, 1981). Esta disposición estructural, tan influyente en el territorio gallego, supone que muchas de las variables relacionadas con la superficie de la cuenca vertiente no presenten un comportamiento lineal. En el caso del río Cabe, por ejemplo, muchas de las mayores anchuras de cauce se alcanzan en el sector central de la cuenca, coincidiendo con la ULT D1.

\section{Conclusiones}

Los dos grandes procedimientos estadísticos manejados (ANOVA y de clasificación / factorial) reflejan cómo las variables de contraste son capaces de independizar las ULT previamente definidas, lo que permite zonificar el territorio en sectores que contengan un comportamiento geomorfológico similar. El análisis ANOVA corrobora como las ULT son diferentes para cada variable, por tanto, con un comportamiento independiente de las restantes. La clasificación en grupos homogéneos (cluster) y el análisis de componentes principales demuestran que las variables de contraste se acoplan bastante bien a la que, teóricamente, debería ser su ULT. De este modo, las variables de contraste también se puedan emplear para definir las características de cada ULT.

En otra línea de conclusiones, el estudio señala que las variables de contraste con más carga explicativa son la ratio a/p, la potencia específica y el $\mathrm{D}_{50}$ del sedimento. La pendiente por redundancia con la potencia pierde protagonismo, y el radio hidráulico es más dependiente del tamaño de cuenca vertiente que del tipo de ULT. Igualmente, las clases de topografía y litología que conforman una ULT influyen de modo diferente sobre cada variable de contraste. Las unidades topográficas se relacionan mejor con la tasa de encajamiento ( $\mathrm{a} / \mathrm{p}$ ), la pendiente y la potencia específica (Tabla 5). Las unidades litológicas lo hacen mejor con $\mathrm{D}_{50}$ (Tabla 5). El radio hidráulico no se ajusta claramente a ninguna de las clases, por el hecho, ya comentado, de no ser dependiente del tipo de ULT, sino de la cuenca vertiente.

En suma, las principales conclusiones extraíbles del presente estudio se pueden sintetizar en 5 puntos. i) La inclusión de las ULT fortalece el modelo de caracterización jerárquica de los hábitats fluviales de la DMA. Con esta nueva concepción jerárquica se combina el criterio tamaño de cuenca con los incorporados por la ULT, lo que potencia la capacidad caracterizadora de hábitats fluviales semejantes. Además, el sesgo 
explicativo de un criterio puede ser completado o, cuando menos, aclarado por otros. Por ejemplo, el tipo de ULT ayuda en la comprensión de por qué, para el río Cabe, el tamaño del cauce no es directamente proporcional a la cuenca vertiente o la pendiente se relaciona inversamente con esta. ii) Las ULT funcionan como unidades espaciales con un comportamiento geomorfológico propio, por lo que controlan procesos característicos de los hábitats fluviales a escalas de más detalle. iii) Las ULT permiten, con suma cautela, extrapolar comportamientos geomorfológicos entre mismas ULT inconexas espacialmente. iv) En caso de que se trabajase a nivel de cuenca, es recomendable sopesar la posibilidad de reajustar las ULT, ya que puede suceder que unidades inferiores a $10 \mathrm{~km}^{2}$ sí tengan cabida espacial por su singularidad geomorfológica respecto al territorio circundante. v) Las características de cada ULT requieren un trabajo de campo más intenso y diversificado que, en definitiva, fortalezca la definición geomorfológica de cada ULT mediante aquellas variables que mejor la definan. En este sentido, la vegetación de ribera es un magnífico bioindicador de las particularidades hidrológicas y geomorfológicas que rigen un cauce (REFCOND, 2003), por lo que la biogeografía geomorfológica se puede usar como una herramienta reflejo del ambiente ecológico sobre el que se desenvuelve una especie vegetal concreta (Freire y Guitián, 2005) e, inversamente, a partir de ese ambiente ecológico es posible extraer las condiciones hidrogeomorfológicas presentes en el río.

\section{Agradecimientos}

A Alfredo Ollero y Augusto Pérez-Alberti por todo el apoyo recibido en esta etapa predoctoral. A Askoa Ibisate, Alba Rey, Natalia Bouso, José Francisco Canosa, Estela Nadal-Romero y Jeffrey Evans por sus sugerentes aportaciones técnicas. A Efrén Muñoz, Aníbal Mejuto, Samuel García y David Varela por las fatigadoras jornadas de campo. A la Consellería de Educación e Ordenación Universitaria de la Xunta de Galicia por las ayudas concedidas mediante el Plan Galego de Investigación, Innovación e Crecemento 2011-2015 (Plan I2C) para el año 2011.

\section{Bibliografía}

Angelier, E. (2002) Ecología de las aguas corrientes. Zaragoza, Acribia.

Bagnold, R.A. (1960) Sediment discharge and stream power - a preliminary announcement. U.S. Geol. Surv., Circular 421, 1-23.

Bailey, R.G. (2005) Identifying ecoregion boundaries. Environmental Management, 34(I), $14-26$.
Balce, A. (1987) Determination of optimun sampling interval in grid digital elevation models (DEM) data adquisition. Photogrammetric Engineering and Remote Sensing, 53 (3), 323-330.

Barredo, J.I. y Bosque-Sendra, J. (1996) Delimitación de unidades homogéneas del relieve a partir de un modelo digital de elevaciones. Estudios geográficos, 225, 615-643. 
Brierley, G.J. y Fryirs, K.A. (2005) Geomorphology and river management. Applications of the river styles framework. Oxaford, Blackwell.

Brooks, K.N., Ffolliott, P.F. y Magner, J.A. (2012) Hydrology and the management of watersheds. Chichester, Wiley-Blackwell.

Brussock, P.P., Brown, A.V. y Nixon, J.C. (1985) Channel form and stream ecosystem models. Water Resources Bulletin, 21, 859-866.

Burrough, P.A. y McDonnell, A. (1998) Principles of Geographical Information Systems for land resources assesment. Somerset, Oxford University Press.

Carrión, J. (dir., 2001) Gran Atlas de Lugo. Lugo, Ed. El Progreso.

CEDEX (2004) Caracterización de los tipos de ríos y lagos. Versión 1.0. Madrid, Centro de Estudios Hidrográficos, Ministerio de Fomento.

CEN Commision of the European Communities (2002) A guidance standard for assessing the hydromorphological features of rivers. CEN TC 230/WG 2/TG 5: N32.

Charlton, R. (2007) Fundamentals of fluvial geomorphology. London, Routledge.

Chorley, R.J. (1969) The drainage basin as the fundamental geomorphic unit. En Chorley, R.J. (ed.) Water, Earth and Man, London, Methuen, p. 77-100.

Church, M.A., McLean, D.G y Wolcott, J.F. (1987) River bed gravels: sampling and analysis. En Thorne, C.R., Bathurst, J.C. y Hey, R.D. (eds.) Sediment transport in gravel-bed rivers, Chichester, Wiley, p. 43-88.

De Vicente, G., Vegas, R., Muñoz-Martín, A., Silva, P.G., Andriessen, P., Cloetingh, S., González Casado, J.M., Van Wees, J.D., Álvarez, J., Carbó, A. y Olaiz, A. (2007) Cenozoic thick-skinned deformation and topography evolution of the Spanish Central System. Global and Planetary Change, 58, 335-381.
Downs, P.W. y Brookes, A. (1994) Developing a standard geomorphological approach for the appraisal of river projects. En Kirby, C. y White, W.R. (eds.) Integrated river basin development. Chichester, Wiley, p. 299-310.

Emery, J.C., Gurnell, A.M., Clifford, N.J., Petts, G.E., Morrissey, I.P. y Soar, P.J. (2003) Classifying the hydraulic performance of rifflepool bedforms for habitat assessment and river rehabilitation design. River Research and Applications, 19, 533-549.

Evans, I. (1972) General geomorphometry, derivatives of altitude, and descriptive statistics. En Chorley, R.J. (ed.) Spatial Analysis in Geomorphology. London, Methuen, p. 17-90.

Felicísimo, A.M. (1994) Modelos digitales del terreno. Oviedo, Pentalfa Ediciones.

Fernández, J.A., Martínez, C. y Magdaleno, F. (2012) Application of indicators of hydrologic alterations in the designation of heavily modified water bodies in Spain. Environmental Science \& Policy, 16, 31-43.

Freire, M.A. y Guitián, L. (2005) Caracterización de la vegetación en los ambientes ribereños del curso medio del Ulla. Xeográfica, 5, 61-83.

Frissell, C., Liss, W.J., Warren, C.E. y Hurley, M.D. (1986) A hierarchical framework for stream hábitat classification: viewing streams in a watershed context. Environmental Management, 10(2), 199-214.

Fryirs, K.A. y Brierley, G.J. (2012) Geomorphic analysis of river systems: An approach to reading the landscape. Oxford, Wiley-Blackwell.

García, C. (1994) Métodos para la caracterización granulométrica en ríos de gravas. Notes de Geografía Física, 23, 5-16.

González del Tánago, M. y García de Jalón, D. (2006) Propuesta de caracterización jerárquica de los ríos españoles para su clasificación según la Directiva Marco del Agua. Limnética, 25(3), 693-721. 
González del Tánago, M. y García de Jalón, D. (2007) Restauración de ríos. Guía metodológica para la elaboración de proyectos. Madrid, Ministerio de Medio Ambiente, Madrid.

Goodwin, C.N. (1999) Fluvial classification: neanderthal necessity or needless normalcy. En Olson, D.S. y Potyondy, J.P. (eds.) Wildland Hydrology. Virginia, American Water Resources Association, p. 229-236.

Gutiérrez Elorza, M. (1994) Introducción a la geomorfología española. En Gutiérrez Elorza, M. (ed.) Geomorfología de España. Madrid, Rueda, p. 1-24.

Hardy, T., Panja, P. y Mathias, D. (2005) WinXSPRO, A channel cross section analyzer, User's manual, version 3.0. Gen. Tech. Rep. RMRS-GTR-147. Fort Collins, U.S. Department of Agriculture, Forest Service, Rocky Mountain Research Station.

Hawkins, C.P., Kershner, J.L., Bisson, P.A., Bryant, M.D., Decker, L.M., Gregory, S.V., McCullough, D.A., Overton, C.K. y Reeves, G.H. (1993) A hierarchical approach to classifying stream habitat features. Fisheries, 18(6), 3-12.

Hawkins, C.P y Norris, R.H. (eds., 2000) Landscape classifications: aquatic biota and bioassessments. Journal of the North American Benthological Society, 19 (3).

Hem, J.D. (1985) Study and interpretation of the chemical characteristics of natural water. Washington, DC: U.S. Geol. Surv. Water Supply Pap. 2254.

Hernández-Pacheco, E. (1934) Sintesis fisiográfica y geológica de España. Trabajos del Museo Nacional de Ciencias Naturales (Serie Geológica) (dos fascículos), 38, Madrid, (volumen correspondiente al año 1932).

Herod, A. (2003) Scale: the local and the global. En Holloway, S.L., Rice, S.P. y Valentine, G. (eds.) Key concepts in Geography. London, SAGE Publications, p. 233-247.
Horacio, J. (2010) Procesamiento de las mallas de cotas altimétricas (MDT25) elaboradas por el Instituto Geográfico Nacional (IGN) para el análisis geoestadístico de los modelos digitales de elevaciones (MDE). En Ojeda, J., Pita, M.F. y Vallejo, I. (eds.) Tecnologias de la Información Geográfica: La Información Geográfica al servicio de los ciudadanos. Secretariado de Publicaciones de la Universidad de Sevilla, p. 172-178.

Horacio, J. y Ollero, A. (2011) Clasificación geomorfológica de cursos fluviales a partir de sistemas de información geográfica (S.I.G.). Boletín de la Asociación Española de Geógrafos, 56, 373-396.

Hupp, C.R. y Osterkamp, W.R. (1996) Riparian vegetation and fluvial geomorphic processes. Geomorphology, 14, 277-295.

Ibisate, A., Ollero, A. y Díaz, E. (2011) Influence of catchment processes on fluvial morphology and river habitats. Limnética, 30(2), 169-182.

Illies, J. y Botosaneanu, L (1963) Problèmes et méthodes de la classification et de la zonation écologiques des eaux courantes, considerées sutout du point de vue faunistique. Mitt. Internat. Verein. Limnol. 19, 1-15.

Jenness, J.S. (2004) Calculating landscape surface area from digital elevation models. Wildlife Society Bulletin, 32(3), 829-839.

Juracek, K. y Fitzpatrick, F. (2003) Limitations and implications of stream classification. Journal of the American Water Resources Association, 39(3), 659-670.

Knighton, D. (1998) Fluvial forms and processes: a new perspective. New York, Oxford University Press.

Kondolf, G.M., Montgomery, D.R., Piégay, H. y Schmitt, L. (2003) Geomorphic classification of rivers and streams. N Kondolf, G.M. y Piégay, H. (eds.) Tools in Fluvial Geomorphology. Chichester, Wiley, p. 171-204. 
Kondolf, M. (1995) Case studies and reviews. Geomorphological stream channel classification in aquatic hábitat restoration: uses and limitations. Aquatic Conservation: $\mathrm{Ma}$ rine and Freshwater Ecosystems, 5, 127-141.

Lara, F., Garilleti, R. y Calleja, J.A. (2007) La vegetación de ribera de la mitad norte española. Madrid, CEDEX Monografías (2 $2^{\underline{a}}$ Ed.), Ministerio de Fomento.

Leopold, L.B. y Maddock, T. (1953) The hydraulic geometry of stream channels and some physiographic implications. U.S. Geological Survey Prof. Paper 252.

Lotspecich, F.B. (1980) Watershed as the basic ecosystem: this conceptual framework provides a basis for a natural classification system. Water Resources Bulletin, 16(4), 581-586.

Malavoi, J.R. y Bravard, J.P. (2010) Éléments d'bydromorphologie fluviale. Vincennes, Office National de l'Eau et des Milieux Aquatiques (ONEMA).

Martín-Serrano, A. (1989) Rasgos generales y problemática de las superficies de erosión en Galicia. Cuad. Lab. Xeol. Laxe, 14, 7-18.

Martín-Serrano, A. (1991) La definición y el encajamiento de la red fluvial actual sobre el macizo herpérico en el marco de su geodinámica alpina. Rev. Soc. Geol. España, 4(3-4), 337-351.

Martín-Serrano, A. (1994) El relieve del Macizo Hespérico: génesis y cronología de los principales elementos morfológicos. Cuad. Lab. Xeol. Laxe, 19, 37-55.

Martín-Vide, J.P. (2002) Ingeniería de ríos. Barcelona, Universitat Politècnica de Catalunya.

Montgomery, D.R. (1996) Influence of geological processes on ecological systems. En Schoonmaker, P.K. von Hagen, B. y Wolf, E.C. (eds.) The rain forests of home: portrait of a North American bioregion. Island Press, p. 43-68.
Montgomery, D.R. (1999) Process domains and the river continuum. Journal of the American Water Resources Association, 35, 397410 .

Montgomery, D.R. y Buffington, J.M. (1998) Channel processes, classification, and response. En Naiman, R.J. y Bilby, R.E. (eds,) River ecology and management: lessons from the Pacific Coastal ecoregion, New York, Springer, p. 13-42.

Moore, I.D., Grayson, R.B. y Landson, A.R. (1991) Digital Terrain Modelling: a review of hydrological, geomorphological, and biological applications. Hydrological Processes, 5(1), 3-30.

Mosley, M.P. (1987) The classification and characterization of rivers. En Richards, K. (ed.) River channels, environmment and process. Oxford, Blackwell, p. 295-320.

Naiman, R.J. (1998) Biotic stream classification. En Naiman, R.J. y Bilby, R.E. (eds,) River ecology and management: lessons from the Pacific Coastal ecoregion, New York, Springer, p. 97-119.

Naiman, R.J., Lonzarich, D.G., Beechie, T.J. y Ralph, S.C. (1992) General principles of classification and the assessment of conservation pontencial in rivers. En Boon, P.J., Calow, P. y Petts, G.E. (eds.) River conservation and management. Chichester, Wiley p. 93-124.

Newson, M.D. (2002) Geomorphological concepts and tools for sustainable river ecosystem management. Aquatic Conservation: Marine and Freshwater Ecosystems, 12, 365-379.

Newson, M.D. (2012) From channel to catchment: a 20-year journey for river management in England and Wales. En Boon, P.J., Calow, P. y Petts, G.E. (eds.) River conservation and management. Chichester, Wiley p. $17-27$. 
Newson, M.D., Clark, M.J., Sear, D.A. y Brookes, A. (1998) The geomorphological basis for classifying rivers. Aquatic Conservation: Marine and Freshwater Ecosystems, 8, 415-430.

Ollero, A. (2011) Los cauces fluviales como indicadores de cambio global: propuesta metodológica. Zubia, monográfico 23, 187-200.

Ollero, A., Ballarín, D., Domenech, S., Mora, D. y Sánchez Fabre, M. (2010) La Directiva Marco del Agua (2000/60/CE) y los ríos en roca. En Ortega, J.A. y Durán, J.J. (eds.) Patrimonio geológico: los ríos en roca de la Peninsula Ibérica, Madrid, Instituto Geológico y Minero de España, p. 163-179.

Ollero, A., Echeverría, M.T., Sánchez Fabre, M., Auría Izquierdo, V., Ballarín, D. y Mora, D. (2003) Metodología para la tipificación hidromorfológica de los cursos fluviales de Aragón en aplicación de la Directiva Marco de Aguas (2000/60/CE). Geographicalia, $44,7-25$.

Omernik, J.M. y Bailey, R.G. (1997) Distinguishing between watershed and ecoregions. $J$. Am. Wat. Resour. Assoc., 33, 935-951.

Palmer, M., Menninger, H.L. y Bernhardt, E.S. (2010) River restoration, habitat heterogeneity and biodiversity: a failure of theory or practice? Freshwater Biology, 55(1), 205-222.

Pardo, A. y Ruiz, M.A. (2005) Análisis de datos con SPSS 13 Base. Madrid, McGraw-Hill.

Pennak, R.W. (1971) Toward a classification of lotic hábitats. Hydrobiologica, 38, 321-334.

Pérez-Alberti, A. (1981) Aproximación xeográfica aos vales fluviais en Galicia. Cuadernos de Estudios Galegos, 32(96-97), 7-36.

Pérez-Alberti, A. (1982) Xeomorfoloxía. En Pérez-Alberti, A. (dir.) Xeografia de Galicia. Tomo I. A Coruña, Sálvora, p. 9-69.

Pérez-Alberti, A. (1991) La Geomorfología de la Galicia Sudoriental. Tesis doctoral (in- édita), Universidade de Santiago de Compostela.

Pérez-Alberti, A. (1993) Xeografía de Galicia: xeomorfoloxía. Gran Enciclopedia Galega, Tomo 3 .

Petts, G. E. y Amoros, C. (cords., 1996) Fluvial bydrosystems. London, Chapman \& Hall.

Peuker, T.K. y Douglas, D.H. (1975) Detection of surface-specific points by parallel processing of discrete terrain elevation data. Computer Graphics and Image Processing, 4 (4), 375-387.

Pike, R.J., Evans, I. y Hengl, T. (2008) Geomorphometry: a brief guide. En Hengl, T. y Reuter, H.I. (eds.) Geomorphometry Concepts, Software, Applications. Series Developments in Soil Science - Volume 33, Amsterdam, Elsevier.

Poff, N.L., Allan, J.D., Bain, M.B., Karr, J.R., Prestegaard, K.L., Richter, B.D., Sparks, R.E. y Stromberg, J.C. (1997) The natural flow regime. BioScience, 47(11), 769-784.

Poole, G.C. (2002) Fluvial landscape ecology: addressing uniqueness within the river discontinuum. Freshwater Biology, 47, 641-660.

REFCOND (2003) Guidance on establishing reference conditions and ecological status class boundaries for inland surface waters. EU Common Implementation Strategy for the Water Framework Directive.

Riley, S.J., DeGloria, S.D. y Elliot, R. (1999) A terrain ruggedness index that quantifies topographic heterogeneity. Intermountain Journal of Sciences, 5(1-4), 23-27.

Rivas-Martínez, S., Penas, A. y Díaz, T.E. (2004) Biogeographic Map of Europe (scale: 1. 6.000.000). Cartographic Service, University of León, Spain.

Rodríguez-Guitián y M.A.; Ramil-Rego, P. (2007) Clasificaciones climáticas aplicadas a Galicia: revisión desde una perspectiva biogeográ- 
fica. Recursos Rurais, Revista oficial do Instituto de Biodiversidade Agraria e Desenvolvemento Rural (IBADER), 3, 31-53.

Rubin J. (1967) Optimal classification into groups: An approach for solving the taxonomy problem. Journal of Theoretical Bio$\log y, 15(1), 103-144$.

Sappington, J.M., Longshore, K.M. y Thompson, D.B. (2007) Quantifying landscape ruggedness for animal habitat analysis: a case study using bighorn sheep in the Mojave desert. The Journal of Wildlife Management, 71(5), 1.419-1.426.

Schmitt, L. (2001). Typologie hydro-geomorphologique fonctionnelle de cours d'eau. Recherche methodologique appliquee aux systemes fluviaux d'Alsace. Thèse de Doctoral de l'Université Louis Pasteur, Strasbourg I.

Schmitt, L., Maire, G., Nobelis, P. y Humbert, J. (2007) Quantitative morphodynamic typology of rivers: a methodological study based on the French Upper Rhine basin. Earth Surface Processes and Landforms, 32, 1.726-1.746.

Strahler, A.N. (1964) Quantitative geomorphology of drainage basins and channels networks. En Chow, V.T. (ed.) Handbook of applied hydrology. New York, McGraw Hill, p. 39-76.

Thorne, C.R. (1997) Channel types and morphological classification. En Thorne, C.R., Hey, R.D. y Newson, M.D. (eds.) Applied fluvial geomorphology for river engineering and management. Chichester, Wiley, p. 175-222.
Vidal-Romaní, J.R. (1996) Geomorfología de Galicia. En Rodríguez-Iglesias, F. (ed.) Historia de Galicia. Xeografía Xeral de Galicia. A Coruña, Hércules Ediciones, tomo XVII, p. 36-63.

Wasson, J.G., Chandesris, A., Pella, H. y Blanc, L. (2002) Les hydro-écorégions de France metropolitane. Approche régionale de la typologie des eaux courantes et éléments pour la définition des peuplements de référence d'invertébrés. Programme de Recherche CEMAGREF, Lyon.

Watson, C.C., Biedenharn, D.S. y Thorne, C.R. (1999) Demonstration erosion control. Desing manual. U.S. Army, Engineer Research and Development Center.

Weibel, R. y Heller, M. (1991) Digital Terrain Modelling. En Maguire, D.J., Goodchild, M.F. y Rhind, D.W. (eds.) Geographical Information Systems: principles and applications. London, Longman, 19, 269-297.

Wentworth, C. (1922) A method of measuring and plotting shapes of pebbles. Bulletin of the U.S. Geological Survey, 730, 91-96.

Wolman, M.G. (1954) A method of sampling coarse river-bed material. Transactions of the American Geophysical Union, 35, 951-956.

Wright, J.F., Furse, M.T. y Moss, D. (1998) River classification using invertebrates: RIVPACS applications. Aquatic Conservation: Marine and Freshwater Ecosystems, 8, 617-631.

Zeverbergen, L.W. y Thorne, C.R. (1987) Quantitative analysis of land surface topography. Earth Surface Processes and Landforms, 12(3), 3047-56. 